\title{
Pinning of interfaces in a random elastic medium and logarithmic lattice embeddings in percolation
}

\author{
Patrick W. Dondl \\ Department of Mathematical Sciences, Durham University, \\ Science Laboratories, South Road, Durham DH1 3LE, UK \\ (patrick.dondl@durham.ac.uk)
}

\section{Michael Scheutzow}

Fakultät II, Institut für Mathematik, Sekr. MA 7-5,

Technische Universität Berlin, Strasse des 17 Juni 136, 10623 Berlin,

Germany (ms@math.tu-berlin.de)

\section{Sebastian Throm}

Institut für Angewandte Mathematik, Universität Bonn, Endenicher Allee 60, 53115 Bonn, Germany (throm@iam.uni-bonn.de)

(MS received 31 July 2012; accepted 24 January 2014)

\begin{abstract}
For a model of a driven interface in an elastic medium with random obstacles we prove the existence of a stationary positive supersolution at non-vanishing driving force. This shows the emergence of a rate-independent hysteresis through the interaction of the interface with the obstacles despite a linear (force $=$ velocity) microscopic kinetic relation. We also prove a percolation result, namely, the possibility to embed the graph of an only logarithmically growing function in a next-nearest neighbour site percolation cluster at a non-trivial percolation threshold.
\end{abstract}

\section{Introduction and the main result}

In this paper we consider a model for the propagation of one-dimensional fronts immersed in an elastic medium subject to an external driving force and randomly distributed obstacles. The goal is to understand the overall macroscopic behaviour of such fronts and its dependence on the external forcing. Here we prove existence of stationary solutions at positive driving force, and thus the emergence of hysteresis.

In order to precisely state our model, let $(\Omega, \mathcal{B}, \mathbf{P})$ be a probability space, let $\omega \in$ $\Omega$ and fix $s \in(0,1)$. The random front at time $t$ is given as the graph $(x, u(x, t, \omega))$ of a function $u: \mathbb{R} \times(0, \infty) \times \Omega \rightarrow \mathbb{R}$ solving the semilinear fractional diffusion problem

$$
\left.\begin{array}{rl}
u_{t}(x, t, \omega) & =-(-\Delta)^{s} u(x, t, \omega)-f(x, u(x, t, \omega), \omega)+F, \\
u(x, 0, \omega) & =0 .
\end{array}\right\}
$$

The function $f(x, y, \omega) \geqslant 0$ is assumed to be locally smooth in $x$ and $y$ for any $\omega$ and of the form of localized obstacles of identical shape and random positions with uniform density, i.e. the obstacle centres are given by a two-dimensional Poisson 


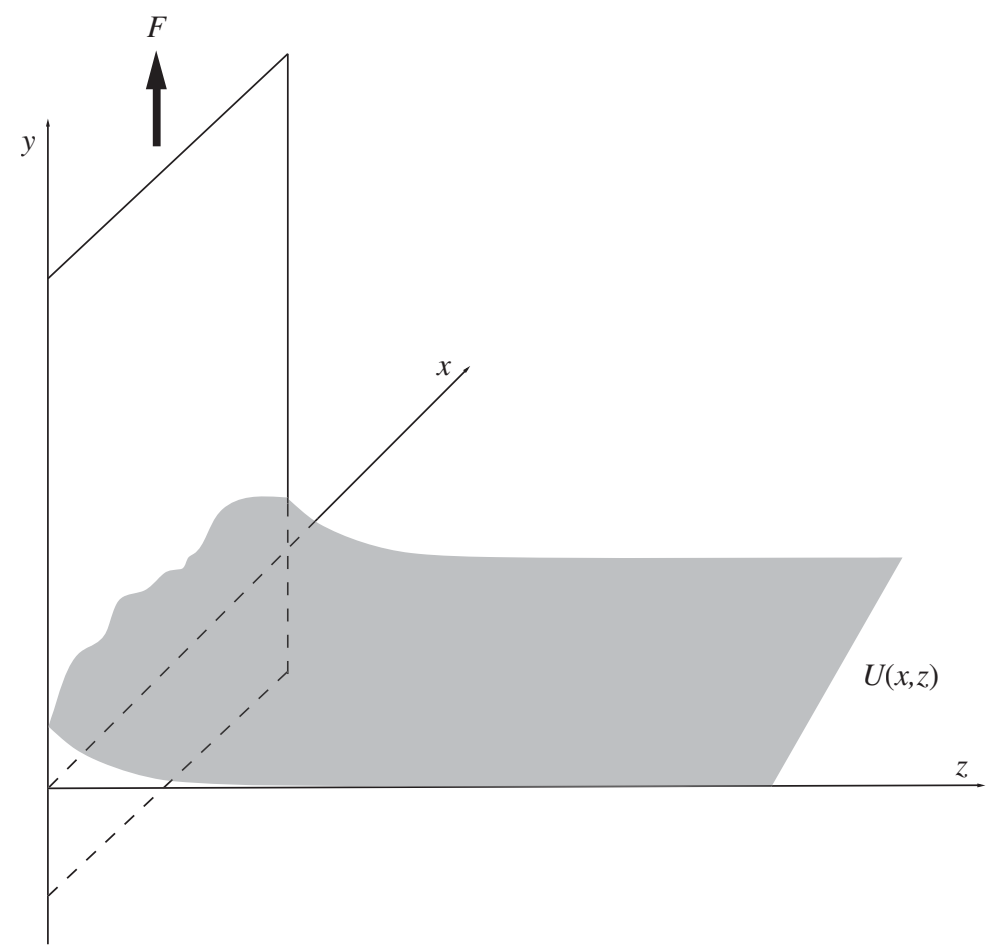

Figure 1. Pulling sandpaper out of water.

process. (See assumption 1.1 for a precise statement.) The constant term $F$ is an external loading and the fractional Laplacian models the interaction of the front with the elastic medium in which it is immersed.

Evolution problems of this kind arise in a large number of physical systems; in particular, the case $s=1 / 2$ is relevant in applications. A simple example is that of pulling sandpaper out of a glass of water. As illustrated in figure 1, we model the evolution of the wetting line of the water surface on a rough plate as the plate gets pulled out of the water. Equation (1.1) can here be formally derived as follows, the derivation in other physical systems (for, example, crack fronts) being similar. We assume the motion of the wetting line $u: \mathbb{R} \rightarrow \mathbb{R}$ to be slow compared to the relaxation time of the water surface $U: \mathbb{R} \times \mathbb{R}^{+} \rightarrow \mathbb{R}$. The system contains an energy term stemming from the water's surface energy, which is given (after removing the constant term from a completely flat surface) as

$$
\int_{\mathbb{R} \times \mathbb{R}^{+}}-1+\sqrt{|\nabla U|^{2}+1} \mathrm{~d} x .
$$

Linearizing this energy around a nearly flat state and cancelling the constant term, we approximate this as

$$
\int_{\mathbb{R} \times \mathbb{R}^{+}} \frac{1}{2}|\nabla U|^{2} \mathrm{~d} x
$$

It is well known that the infimum of this energy subject to the condition that $U=u$ on the boundary of the domain is given by $\frac{1}{2}[u]_{H^{1 / 2}}^{2}$, i.e. the $H^{1 / 2}$-norm squared 
of the function setting the boundary condition. The variation of the $H^{1 / 2}$-norm squared yields the term containing the square root of the Laplacian in (1.1). The constant term $F$ models the constant force with which the rough surface is pulled out of the water. The roughness of the surface itself acts as an obstacle to the evolution of the wetting line: it locally requires an additional amount of force to overcome a grain in the sandpaper. This is modelled by the heterogeneous force term $f$, yielding (1.1) as the viscous flow with respect to the involved force terms. An experimental example of this kind of system can be found in [13]. They use a similar model, which is also proposed in $[7,12]$. The non-local term in these papers is a mean-field version of our term.

Another important application in which a model of the above kind arises is that of a crack front propagating in a rough medium. Experiments and some modelling can be found in the work of Schmittbuhl et al. [14]. The derivation of the stress intensity factor for a non-flat crack front (resulting in the fractional Laplacian) was first given by Gao and Rice [9]; for simulations using the model and for more experimental references see, for example, [15]. Non-local operators that model the interaction with elastic media also arise in models for dislocations $[1,8]$.

In this paper, we consider a specific form of the function $f$, which is that of localized smooth obstacles.

Assumption 1.1 (the random field). Fix $r_{1}, r_{2}>0$ such that $r_{2}>\sqrt{2} r_{1}$. Let $\left\{\left(x_{k}(\omega), y_{k}(\omega)\right)\right\}_{k \in K}$ be a two-dimensional Poisson process on $\mathbb{R} \times\left[r_{1}, \infty\right)$ with intensity $\lambda>0$ and consider the random set

$$
O(\omega)=\bigcup_{k \in K} B_{\left(\sqrt{2} r_{1}+r_{2}\right) / 2}\left(x_{k}, y_{k}\right)
$$

For $q>0$, we take $f$ to be of the form

$$
f(x, y, \omega)=q \eta_{\left(r_{2}-\sqrt{2} r_{1}\right) / 2} * \chi_{O(\omega)},
$$

where $\eta_{\delta}$ is a standard mollifier of radius $\delta$ and $\chi_{O}$ is the characteristic function of the set $O$.

REMARK 1.2. Given assumption 1.1, it is clear that (1.1) admits a unique viscosity solution. See, for example, [6], noting that the right-hand side in our equation is uniformly Lipschitz.

Under these conditions we can state the main theorem of this paper.

THEOREM 1.3 (pinning of interfaces). Assume that the function $f$ is chosen according to assumption 1.1. There then exist a deterministic $F^{*}>0$ and a continuous random function $u: \mathbb{R} \times \Omega \rightarrow[0, \infty)$ with the property that the function $\bar{u}(x, t, \omega):=\min \left(F^{*} t, u(x)\right)$ is a supersolution, according to definition 1.4 , to the evolution problem (1.1) for $F \leqslant F^{*}$ and for almost every $\omega \in \Omega$.

Furthermore, we can choose $u$ such that there exist constants $C>0$ and $q>0$ so that for any $x \in \mathbb{R}$ we have $\mathbf{P}\{u(x)>h\} \leqslant C \mathrm{e}^{-q h}$, i.e. the height of the pinned interface admits an exponential tail in its distribution. In particular, for any $x \in \mathbb{R}$, the expected value of the height of the pinned interface satisfies $\mathbf{E}(u(x))<\beta$ for some fixed $\beta<\infty$, depending only on the deterministic parameters of the obstacle distribution and on $s$. 
In this theorem we use the definition of a supersolution to a fractional diffusion equation as found, for example, in [6]. In the following, we write the fractional Laplacian as $\mathrm{A}:=-(-\Delta)^{s}$. Since $s \in(0,1)$ can be taken as a constant throughout the paper, we do not explicitly denote the dependence of A on $s$. We repeat the definition, adapted to our setting, for the reader's convenience.

Definition 1.4. Fix $T>0$. A bounded lower semi-continuous function $u: \mathbb{R} \times$ $[0, T) \rightarrow \mathbb{R}$ is a viscosity supersolution to $(1.1)$ if $u(\cdot, 0) \geqslant 0$ and if, for all $(x, t) \in$ $\mathbb{R} \times(0, T)$ and all $(\alpha, p) \in \mathbb{R} \times \mathbb{R}$ such that there exists $\sigma>0$ and $r>0$ satisfying

$$
u(y, s) \geqslant u(x, t)+\alpha(\tau-t)+p(y-x)-\sigma|y-x|^{2}+o(\tau-t)
$$

for $y \in B_{r}(x)$ and $\tau \in[0, T)$, we have

$$
\alpha \geqslant \mathrm{A} u(x, t)-f(x, u(x, t))+F .
$$

REMARK 1.5. By the comparison principle, of course any random field that can be bounded from below for almost every $\omega$ by a field of the type of assumption 1.1 yields the same pinning result. Theorem 1.3 is thus valid for a whole class of models, as long as a viscosity solution to the model exists.

REMARK 1.6. We will equivalently refer to the stationary function $u$ as the supersolution, since $\bar{u}$ is bounded by $u$ from above for all times.

The physical interpretation of our result is thus the following. Theorem 1.3 states that there is a non-trivial pinning threshold in our model, since by the comparison principle (see, for example, [11, theorem 2]), any solution of the fractional diffusion problem (1.1) with $F \leqslant F^{*}$ and zero initial condition must remain below the nonnegative supersolution $u$ for all times. Note that assumption 1.1 ensures that an identically zero function is a stationary subsolution to the evolution problem (1.1) for any $F \geqslant 0$. Thus, for $0 \leqslant F \leqslant F^{*}$, the interface becomes trapped and reaches, at least asymptotically, a stationary state. The estimate on the expectation of $u$ (together with ergodicity) shows that the area (per unit length) swept out by the interface is bounded.

The paper is organized as follows. In $\S 2$ we show the existence of a non-trivial threshold for the existence of infinite percolation clusters that contain the graph of a function that only grows logarithmically. This is a generalization of Lipschitz percolation [4] and the proof for our result is inspired by [10]. In $\S 3$, using the percolation result, the supersolution is constructed. In contrast to [5], due to the non-local nature of the problem, a simple piecewise construction is no longer sufficient. Finally, in $\S 4$ we present some conclusions and open problems.

\section{Flat percolation clusters}

In this section, let $\|\cdot\|$ denote the $l_{1}$-norm on $\mathbb{Z}^{n}$ and denote the $i$ th canonical unit vector in $\mathbb{Z}^{n+1}$ by $e_{i}(1 \leqslant i \leqslant n+1)$. The non-negative integers are denoted by $\mathbb{N}_{0}$. We will also use the notation for multinomial coefficients

$$
\left(\begin{array}{c}
m \\
k_{1}, k_{2}, \ldots, k_{r}
\end{array}\right)=\frac{m !}{k_{1} ! k_{2} ! \cdots k_{r} !} \quad \text { with } \sum_{i=1}^{r} k_{i}=m .
$$


TheOREM 2.1. Consider site percolation on $\mathbb{Z}^{n+1}$ with $n \geqslant 1$ : let $(\bar{\Omega}, \overline{\mathcal{B}}, \overline{\mathbf{P}})$ be a probability space and assume that for given $p \in[0,1]$ the $\{0,1\}$-valued random variables $X_{z}, z \in \mathbb{Z}^{n+1}$ are independent and identically distributed with $\overline{\mathbf{P}}\left\{X_{z}=\right.$ $1\}=p$. We say that site $z \in \mathbb{Z}^{n+1}$ is open if $X_{z}(\omega)=1$, otherwise it is closed. For each non-decreasing function $H: \mathbb{N}_{0} \rightarrow \mathbb{N}_{0}$ satisfying:

(i) $H(0)=0$,

(ii) $H(1) \geqslant 1$,

(iii) $\liminf _{k \rightarrow \infty} \frac{H(k)}{\log k}>0$,

there exists some $p_{H}=p_{H}(n) \in(0,1)$ such that for each $p \in\left(p_{H}, 1\right)$ there exists a set $\bar{\Omega}_{0} \subseteq \bar{\Omega}$ of full measure such that there exists a function $\Lambda: \bar{\Omega} \times \mathbb{Z}^{n} \rightarrow \mathbb{N}$ that satisfies the following statements.

(a) For all $x, y \in \mathbb{Z}^{n}$ and $\omega \in \bar{\Omega}_{0}$, we have that $|\Lambda(\omega, x)-\Lambda(\omega, y)| \leqslant H(\|x-y\|)$.

(b) For every $x \in \mathbb{Z}^{n}$ and $\omega \in \bar{\Omega}_{0}$ we have that $(x, \Lambda(\omega, x))$ is open.

Proof. It suffices to prove the theorem for the case in which $H(k+1) \leqslant H(k)+$ 1 for all $k \in \mathbb{N}_{0}$ (which implies that $H(1)=1$ ). Furthermore, we can and will assume without loss of generality that for all positive integers $k_{1}, \ldots, k_{m}$, we have $H\left(\sum_{j} k_{j}\right) \leqslant \sum_{j} H\left(k_{j}\right)$.

For $j \in \mathbb{N}$ define

$$
R(j):=\sup \{k \in \mathbb{N}: H(k)=j\} .
$$

Now we explain what we mean by an admissible path. Let $u, v \in \mathbb{Z}^{n+1}$. We define a blocking-path from $u$ to $v$ to be any finite sequence of distinct sites $u=$ $u_{0}, u_{1}, \ldots, u_{k}=v$ in $\mathbb{Z}^{n+1}$ such that for each $i=1, \ldots, k$ the difference $u_{i}-u_{i-1}$ takes either the value $e_{n+1}$ or $(y,-H(\|y\|))$ for some $y \in \mathbb{Z}^{n} \backslash\{0\}$. For a given $\omega \in \bar{\Omega}$, a blocking-path is called admissible if, in addition, for each $i=1, \ldots, k$ we have that if $u_{i}-u_{i-1}=e_{n+1}$, then $u_{i}$ is closed, i.e. $X_{u_{i}}(\omega)=0$. The relevance of such admissible blocking paths in the sense of theorem 2.1 is the following. If for given $\omega \in \bar{\Omega}$ there exists an admissible blocking path from any site $(y, 0), y \in \mathbb{Z}^{n}$, to a site $(x, h)$, then every function $\Lambda$ satisfying (a) and (b) from theorem 2.1 must necessarily lie above the admissible path. In the following, however, we will show that if the reachable set of such admissible blocking paths has finite height everywhere, then our function $\Lambda$ is indeed found.

Thus, for $x \in \mathbb{Z}^{n}, \omega \in \bar{\Omega}$, define

$\Lambda(\omega, x):=1+\sup \left\{h \in \mathbb{N}_{0}:\right.$ there exist $y \in \mathbb{Z}^{n}$ and an admissible blocking path from $(y, 0)$ to $(x, h)\}$.

If $\Lambda(\omega, x)$ is finite for some $x \in \mathbb{Z}^{n}$, then $\Lambda\left(\omega, x+e_{i}\right) \leqslant \Lambda(\omega, x)+H(1)$ for each $i \in$ $\{1, \ldots, n\}$ since otherwise there would exist an admissible blocking path starting at some $(y, 0)$, going through $\left(x+e_{i}, h\right)$ for some $h \geqslant \Lambda(\omega, x)+H(1)$ and continuing to (and ending at) $(x, h-H(1))$, thus contradicting the definition of $\Lambda(\omega, x)$. Therefore, $\Lambda(\omega, x)<\infty$ for some $x \in \mathbb{Z}^{n}$ in fact implies that $\Lambda(\omega, x)<\infty$ for all $x \in \mathbb{Z}^{n}$. The 
same argument shows that the function $x \mapsto \Lambda(\omega, x)$ satisfies claim (a) in the statement of the theorem for each $\omega$ for which $\Lambda(\omega, 0)<\infty$.

Clearly, if $\Lambda(\omega, x)$ is finite for some $x \in \mathbb{Z}^{n}$, then $(x, \Lambda(\omega, x))$ is also open. All that remains to be shown is that there exists a set $\bar{\Omega}_{0}$ of full measure such that $\Lambda(\omega, 0)<\infty$ for all $\omega \in \bar{\Omega}_{0}$.

By assumption, there exist $C>0$ and $\gamma>0$ such that $R(i) \leqslant C \mathrm{e}^{\gamma i}$ for all $i \in \mathbb{N}$. Observe that there exists some $K$ such that the number of sites in $\mathbb{Z}^{n}$ with $l_{1}$-norm at most $k$ is bounded by $K k^{n}$ for all $k \in \mathbb{N}$. Fix $N \in \mathbb{N}_{0}, h \in \mathbb{N}$ and $q:=1-p \in(0,1)$. For $x \in \mathbb{Z}^{n}$ with $\|x\|=N$ we estimate the expected value of the number of admissible blocking paths from $(x, 0)$ to $(0, h)$ as follows.

For a given such admissible blocking path, let $k_{i}$ be the number of steps of the path containing a down-jump of size $i \in \mathbb{N}$. Then the expected number of such admissible blocking paths $\left(u_{j}\right)_{j=0}^{k}$ that contain exactly $D \in \mathbb{N}_{0}$ down-steps (in the sense that $\left.\sum_{i=1}^{k}\left(e_{n+1} \cdot\left(u_{i}-u_{i-1}\right)\right)^{-}=D\right)$ and therefore $D+h$ up-steps is at most

$$
\begin{aligned}
\sum\left(\begin{array}{c}
k_{1}+\cdots \\
k_{1}, \ldots
\end{array}\right. & \left.k_{D}, D+h+h\right) k^{D+h} \prod_{i=1}^{D}\left(K R(i)^{n}\right)^{k_{i}} \\
& \leqslant q^{D+h} \mathrm{e}^{\gamma n D} \sum\left(\begin{array}{c}
k_{1}+\cdots+k_{D}+D+h \\
k_{1}, \ldots, k_{D}, D+h
\end{array}\right)\left(K C^{n}\right)^{k_{1}+\cdots+k_{D}} \\
& \leqslant q^{D+h} \mathrm{e}^{\gamma n D} \sum_{m=0}^{D}\left(K C^{n}\right)^{m} \sum\left(\begin{array}{c}
m+D+h \\
k_{1}, \ldots, k_{D}, D+h
\end{array}\right) \\
& =q^{D+h} \mathrm{e}^{\gamma n D} \sum_{m=0}^{D}\left(K C^{n}\right)^{m}\left(\begin{array}{c}
m+D+h \\
D+h
\end{array}\right) \sum\left(\begin{array}{c}
m \\
k_{1}, \ldots, k_{D}
\end{array}\right) \\
& \leqslant q^{D+h} \mathrm{e}^{\gamma n D} \sum_{m=0}^{D}\left(K C^{n}\right)^{m} 2^{m+D+h} 2^{D}\left(\begin{array}{c}
m+D-1 \\
m
\end{array}\right) \\
& \leqslant q^{D+h} \mathrm{e}^{\gamma n D} 2^{2 D+h}\left(\left(2 K C^{n}\right) \vee 1\right)^{D} \sum_{m=0}^{D}\left(\begin{array}{c}
m+D-1 \\
m
\end{array}\right) \\
& =q^{D+h} \mathrm{e}^{\gamma n D} 2^{2 D+h}\left(\left(2 K C^{n}\right) \vee 1\right)^{D}\left(\begin{array}{c}
2 D \\
D
\end{array}\right) \\
& \leqslant q^{D+h} \mathrm{e}^{\gamma n D} 2^{2 D+h}\left(\left(2 K C^{n}\right) \vee 1\right)^{D} 2^{2 D},
\end{aligned}
$$

where the first two sums are extended over all $k_{1}, \ldots, k_{D} \in \mathbb{N}_{0}$ satisfying $\sum_{i=1}^{D} i k_{i}=$ $D$ and the fourth and sixth sums extend over all $k_{1}, \ldots, k_{D} \in \mathbb{N}_{0}$, which in addition satisfy $\sum_{i=1}^{D} k_{i}=m$. Let

$$
\beta:=16 \mathrm{e}^{\gamma n}\left(\left(2 K C^{n}\right) \vee 1\right) .
$$

Summing over $D$ from $H(N)$ to $\infty$, we see that for $q \beta<1$ the expected number of admissible blocking paths from a given point $(x, 0)$ to $(0, h)$ is at most $(2 q)^{h}(q \beta)^{H(N)}(1-q \beta)^{-1}$. The total expected number of admissible blocking paths starting from any point $(y, 0), y \in \mathbb{Z}^{n}$, and ending at $(0, h)$ is thus bounded by

$$
(2 q)^{h}(1-q \beta)^{-1} \sum_{N=0}^{\infty}\left(\left(\left(\tilde{K} N^{n-1}\right) \vee 1\right)(q \beta)^{H(N)}\right) .
$$


Here, $\tilde{K}$ is a constant chosen such that the number of $y \in \mathbb{Z}^{n}$ such that $\|y\|=N$ is bounded by $\tilde{K} N^{n-1}$ for all $N \in \mathbb{N}$. The sum is clearly finite provided that $q>0$ is sufficiently small. Now, the first Borel-Cantelli lemma implies that the largest $h$ for which there exists an admissible blocking path from some $(y, 0)$ and ending at $(0, h)$ is finite almost surely (i.e. is finite on a set $\bar{\Omega}_{0} \subset \bar{\Omega}$ of full measure), and therefore the assertion is proved.

REMARK 2.2. The theorem is sharp in the sense that it becomes wrong if in (iii) 'inf' is dropped and ' $>$ ' is replaced by ' $=$ ' since in this case the second BorelCantelli lemma shows that for each $m \in \mathbb{N}$ the number of sites $x \in \mathbb{Z}^{n}$ for which all sites $(x, h), h \in\{0, \ldots, H(x)+m\}$, are closed is almost surely infinite (and hence strictly positive). This implies that for every $m \in \mathbb{N}$, the set of all $\omega \in \bar{\Omega}$ for which there exists a function $\Lambda: \mathbb{Z}^{n} \rightarrow \mathbb{N}$ satisfying $\Lambda(0) \leqslant m$ and (a) and (b) in theorem 2.1 has measure zero. Hence, the set of all $\omega \in \bar{\Omega}$ for which there exists a function $\Lambda: \mathbb{Z}^{n} \rightarrow \mathbb{N}$ satisfying (a) and (b) in theorem 2.1 has measure zero.

REMARK 2.3. Define $p_{H}(n)$ as in the proof of theorem 2.1, i.e. $p_{H}(n)$ is the supremum over all $p$ such that $(1-p) \beta<1$ and the sum in $(2.1)$ converges. Let $p>p_{H}(n)$ and let $\Lambda: \mathbb{Z}^{n} \rightarrow \mathbb{N}$ be the smallest function satisfying (a) and (b) of theorem 2.1. For notational convenience we drop the dependence on $\omega$ here. Furthermore, denote the factor behind $(2 q)^{h}$ in $(2.1)$ by $C_{n, p, H}$. We then get for $m \in \mathbb{N}_{0}$ and $q:=1-p$,

$$
\begin{aligned}
\overline{\mathbf{P}}\{\Lambda(0)>m\}=\sum_{h=m}^{\infty} \overline{\mathbf{P}}\{\Lambda(0)=h+1\} & \leqslant C_{n, p, H} \sum_{h=m}^{\infty}(2 q)^{h} \\
& =C_{n, p, H}(2(1-p))^{m} \frac{1}{1-2 q}
\end{aligned}
$$

so $\Lambda(0)$ has exponential tails. Note that the exponential decay rate does not go to zero as $p$ approaches $p_{H}(n)$ (but $C_{n, p, H}$ blows up).

\section{Construction of the supersolution}

The construction of the supersolution is performed in a series of steps. We first split up $\mathbb{R}^{2}$ into boxes large enough that boxes that contain an obstacle of a minimum strength percolate in the sense of $\S 2$. All obstacles not necessary for the percolation cluster are then disregarded. In each column of boxes we now have one obstacle at position $\left(x_{i}, y\left(x_{i}\right)\right)$. Starting from a periodic supersolution (assuming obstacles at $y=0$ and at periodic distance in $x$ with period larger than the box size), we construct a supersolution for obstacles centred at $\left(x_{i}, 0\right)$ by cutting out one period and using this function locally around obstacle sites. Finally, we can add a smooth function with less than linear growth (given by the percolation cluster) in order to obtain a supersolution that passes through the original obstacle sites.

In this section, we make frequent use of the equivalence of the integral representation and the Fourier representation of the fractional Laplacian. Furthermore, we use the symmetry of the fractional Laplace operator and the weak form of it by switching between applying it to a test function and the function itself. Further information can be found in [3]. The extension problem related to fractional Laplacians has been treated in [2]. 


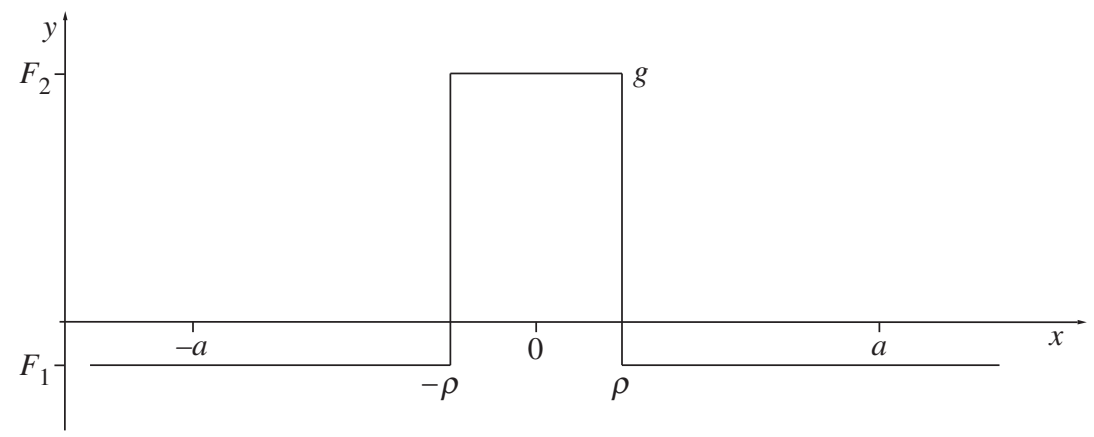

Figure 2. The function $g$.

Definition 3.1. For parameters $a, \rho, F_{2}>0$ with $a>2 \rho$ (which will be fixed later) let $F_{1}:=\rho F_{2} /(a-\rho)$. We then define

$$
g(x):= \begin{cases}F_{2} & \text { for } x \in[-\rho, \rho] \\ -F_{1} & \text { for } x \in[-a, a] \backslash[-\rho, \rho]\end{cases}
$$

while $g$ is extended $2 a$-periodically to $\mathbb{R}$.

Note that the average of $g$ vanishes. We now construct a periodic supersolution.

Definition 3.2. Let $v$ be the (modulo some constant) unique periodic solution of

$$
\mathrm{A} v=g .
$$

To render the solution unique we choose the constant such that the average of $v$ vanishes.

From the eigenvalue representation of A on periodic functions, we can compute the Fourier series representation of $v$, which is then used to compute $L^{\infty}$-bounds and some symmetry properties for $v$.

Lemma 3.3. The Fourier series representation of $v$ is given by

$$
v(x)=-2 a^{2 s}\left(\sum_{k=1}^{\infty} \frac{F_{1}+F_{2}}{\pi^{1+2 s}} \sin \left(k \frac{\pi}{a} \rho\right) \frac{\cos (k \pi x / a)}{k^{1+2 s}}\right) .
$$

Proof. As $g$ is an even function, it suffices to consider only the even eigenfunctions of $-\Delta$ on the interval $(-a, a)$ with periodic boundary condition. We thus take $\varphi_{k}(x)=$ $(1 / \sqrt{a}) \cos (k \pi x / a)$ with associated eigenvalues $\lambda_{k}=k^{2} \pi^{2} / a^{2}$ of the Laplacian.

A simple calculation shows that the representation of $g$ as a Fourier series is given by

$$
g(x)=2 \sum_{k=1}^{\infty} \frac{F_{1}+F_{2}}{k \pi} \sqrt{a} \sin \left(k \frac{\pi}{a} \rho\right) \varphi_{k}
$$


Let $v=\sum_{k=0}^{\infty} \beta_{k} \varphi_{k}$ be the Fourier series representation of $v$ and denote the Fourier coefficients of $g$ by $\alpha_{k}, k=1, \ldots, \infty$. We then have

$$
\mathrm{A} v(x)=-\sum_{k=1}^{\infty} \lambda_{k}^{s} \alpha_{k} \varphi_{k}(x)=g(x),
$$

and so by comparing coefficients we get

$$
\begin{aligned}
\beta_{k} & =-\frac{1}{\lambda_{k}^{s}} \alpha_{k} \\
& =-\frac{1}{\left(k^{2} \pi^{2} / a^{2}\right)^{s}}\left(2 \frac{F_{1}+F_{2}}{k \pi} \sqrt{a} \sin \left(k \frac{\pi}{a} \rho\right)\right) \\
& =-\frac{2}{k^{2 s}}\left(\frac{a}{\pi}\right)^{2 s}\left(\frac{F_{1}+F_{2}}{k \pi} \sqrt{a} \sin \left(k \frac{\pi}{a} \rho\right)\right) .
\end{aligned}
$$

This yields (modulo the constant average of $v$ that we assumed to be zero)

$$
\begin{aligned}
v(x) & =-2\left(\frac{a}{\pi}\right)^{2 s} \sum_{k=1}^{\infty} \frac{1}{k^{2 s}}\left(\frac{F_{1}+F_{2}}{k \pi} \sqrt{a} \sin \left(k \frac{\pi}{a} \rho\right)\right) \\
& =-2 a^{2 s} \sum_{k=1}^{\infty} \frac{F_{1}+F_{2}}{\pi^{1+2 s}} \sin \left(k \frac{\pi}{a} \rho\right) \frac{\cos (k \pi x / a)}{k^{1+2 s}} .
\end{aligned}
$$

REMARK 3.4. Note that by the Fourier characterization of $H_{\text {per }}^{2 s}(Y)$ with $Y=$ $(-a, a)$, we have $v \in H_{\text {per }}^{2 s}(Y)$.

As mentioned before, we can now prove some properties of $v$.

LEMMA 3.5. We have the following:

(i) $v(-x)=v(x)$ for all $x \in \mathbb{R}, v$ is periodic with period $2 a$ and continuous;

(ii) $\|v\|_{L^{\infty}} \leqslant \frac{2\left(F_{1}+F_{2}\right)}{\pi^{2 s}} \zeta(2 s) a^{2 s-1} \rho$ for $s>1 / 2$;

(iii) $\|v\|_{L^{\infty}} \leqslant \frac{2\left(F_{1}+F_{2}\right)}{\pi} \rho(2+\log (a)-\log (\pi \rho))$ for $s=1 / 2$;

(iv) $\|v\|_{L^{\infty}} \leqslant \frac{F_{1}+F_{2}}{s(1-2 s) \pi} \rho^{2 s}$ for $s<1 / 2$.

Here, $\zeta$ denotes the Riemann zeta function.

Proof. (i) The symmetry and periodicity properties follow directly from the corresponding properties of $\cos (\cdot)$. The continuity of $v$ follows from the uniform convergence of its Fourier series. 
(ii) Using the representation

$$
v(x)=-2 a^{2 s} \sum_{k=1}^{\infty} \frac{F_{1}+F_{2}}{\pi^{1+2 s}} \sin \left(k \frac{\pi}{a} \rho\right) \frac{\cos (k \pi x / a)}{k^{1+2 s}}
$$

from lemma 3.3 together with

$$
\left|\sin \left(k \frac{\pi}{a} \rho\right)\right| \leqslant k \frac{\pi}{a} \rho \text { and }\left|\cos \left(k \frac{\pi}{a} x\right)\right| \leqslant 1,
$$

as $k \pi b / a \geqslant 0$, we get

$$
\begin{aligned}
\left|\sum_{k=1}^{\infty} \frac{F_{1}+F_{2}}{\pi^{1+2 s}} \sin \left(k \frac{\pi}{a} \rho\right) \frac{\cos (k \pi x / a)}{k^{1+2 s}}\right| & \leqslant \sum_{k=1}^{\infty} \frac{F_{1}+F_{2}}{\pi^{1+2 s}} \frac{k \pi \rho}{a} \frac{1}{k^{1+2 s}} \\
& =\frac{F_{1}+F_{2}}{\pi^{2 s}} \frac{\rho}{a} \sum_{k=1}^{\infty} \frac{1}{k^{2 s}} \\
& =\frac{F_{1}+F_{2}}{\pi^{2 s}} \frac{\rho}{a} \zeta(2 s) .
\end{aligned}
$$

Finally, this gives

$$
\|v\|_{L^{\infty}} \leqslant 2 a^{2 s}\left(\frac{F_{1}+F_{2}}{\pi^{2 s}} \frac{\rho}{a} \zeta(2 s)\right)=2 \frac{F_{1}+F_{2}}{\pi^{2 s}} \zeta(2 s) a^{2 s-1} \rho .
$$

(iii) For $s=1 / 2$ we have

$$
\begin{aligned}
|v| & =\left|-2 a \sum_{k=1}^{\infty} \frac{F_{1}+F_{2}}{\pi^{2}} \sin \left(k \frac{\pi}{a} \rho\right) \frac{\cos (k \pi x / a)}{k^{2}}\right| \\
& =\frac{2\left(F_{1}+F_{2}\right)}{\pi^{2}} a\left|\sum_{k=1}^{\infty} \frac{\sin (k \pi \rho / a) \cos (k \pi x / a)}{k^{2}}\right| .
\end{aligned}
$$

With $|\sin (k \pi \rho / a)| \leqslant k \pi \rho / a,|\cos (k \pi x / a)| \leqslant 1$ and $|\sin (k \pi \rho / a)| \leqslant 1$, by splitting the sum into two parts and using an integral estimate for each part one gets

$$
\begin{aligned}
|v(x)| & \leqslant \frac{2\left(F_{1}+F_{2}\right)}{\pi^{2}} a\left(\frac{\pi}{a} \rho+\int_{1}^{a / \pi \rho} \frac{\pi}{a} \rho \frac{1}{k} \mathrm{~d} k+\int_{a / \pi \rho}^{\infty} \frac{1}{k^{2}} \mathrm{~d} k\right) \\
& =\frac{2\left(F_{1}+F_{2}\right)}{\pi^{2}}\left(\pi \rho\left(1+\log \left(\frac{a}{\pi \rho}\right)\right)+\frac{a}{a / \pi \rho}\right) \\
& =\frac{2\left(F_{1}+F_{2}\right)}{\pi^{2}}(\pi \rho(2+\log (a)-\log (\pi \rho))) \\
& =\frac{2\left(F_{1}+F_{2}\right)}{\pi} \rho(2+\log (a)-\log (\pi \rho)) .
\end{aligned}
$$


(iv) Similarly, for $s<1 / 2$ we have

$$
\begin{aligned}
|v| & \leqslant 2 \frac{F_{1}+F_{2}}{\pi^{1+2 s}} a^{2 s}\left(\frac{\pi}{a} \rho+\int_{1}^{a / \pi \rho} \frac{\pi}{a} \rho \frac{1}{k^{2 s}} \mathrm{~d} k+\int_{a / \pi \rho}^{\infty} \frac{1}{k^{1+2 s}} \mathrm{~d} k\right) \\
& =2 \frac{F_{1}+F_{2}}{\pi^{1+2 s}} a^{2 s}\left(\frac{\pi}{a} \rho+\frac{\pi \rho}{a(1-2 s)}\left(\left(\frac{a}{\pi \rho}\right)^{1-2 s}-1\right)+\frac{1}{2 s}\left(\frac{a}{\pi \rho}\right)^{-2 s}\right) \\
& =2 \frac{F_{1}+F_{2}}{\pi^{1+2 s}}\left(\frac{\pi \rho}{a^{1-2 s}}\left(1-\frac{1}{1-2 s}\right)+\frac{(\pi \rho)^{2 s}}{1-2 s}+\frac{(\pi \rho)^{2 s}}{2 s}\right) \\
& =2 \frac{F_{1}+F_{2}}{\pi^{1+2 s}}\left(\frac{\pi \rho}{a^{1-2 s}}\left(1-\frac{1}{1-2 s}\right)+(\pi \rho)^{2 s}\left(\frac{1}{1-2 s}+\frac{1}{2 s}\right)\right) \\
& =2 \frac{F_{1}+F_{2}}{\pi^{1+2 s}}\left(-\frac{2 s}{1-2 s} \frac{\pi \rho}{a^{1-2 s}}+\frac{(\pi \rho)^{2 s}}{2 s(1-2 s)}\right) \\
& \leqslant \frac{1}{s(1-2 s) \pi} \rho^{2 s}\left(F_{1}+F_{2}\right) .
\end{aligned}
$$

For the last inequality we used $-(2 s /(1-2 s))\left(\pi \rho / a^{1-2 s}\right) \leqslant 0$ as $s<1 / 2$.

REMARK 3.6. We assumed that $a / \pi \rho>1$ in the proof above as it turns out later (when the parameters are fixed) that $\rho<a / 18$.

Next we will show some monotonicity properties of $v$.

Lemma 3.7. The function $v$ strictly increases on $[0, a]$ (and thus, by symmetry, strictly decreases on $[-a, 0])$.

We denote by $E^{+}:=\bigcup_{k=-\infty}^{\infty}(2 k a-\rho, 2 k a+\rho)$ the set where $g$ is positive and by $E^{-}:=\mathbb{R} \backslash \bar{E}^{+}$the set where $g$ is negative. With this notation we obtain the following proposition.

Proposition 3.8. $(-\Delta)^{p} g(x)$ (given by the integral representation) exists and is continuous on $E^{+} \cup E^{-}$for any $p \in(0,1)$, and one furthermore has

$$
(-\Delta)^{p} g(x) \begin{cases}>0, & x \in E^{+}, \\ <0, & x \in E^{-} .\end{cases}
$$

Proof. The existence and continuity are obvious as $g$ is piecewise constant and we exclude the points where $g$ jumps. For the positivity, respectively, negativity, take $x \in E^{+}$. One then has

$$
(-\Delta)^{p} g(x)=C_{p} \mathrm{PV} \int_{\mathbb{R}} \frac{g(x)-g(y)}{|x-y|^{1+2 p}} \mathrm{~d} y=C_{p} \mathrm{PV} \int_{\mathbb{R}} \frac{F_{1}+F_{2}}{|x-y|^{1+2 p}} \chi_{E^{-}}(y) \mathrm{d} y>0,
$$

where $\chi_{E^{+}}$is the characteristic function of $E^{+}$and 'PV' denotes the principal value of the integral. For $x \in E^{-}$the same calculation gives

$$
(-\Delta)^{p} g(x)=C_{p} \mathrm{PV} \int_{\mathbb{R}} \frac{g(x)-g(y)}{|x-y|^{1+2 p}} \mathrm{~d} y=-C_{p} \mathrm{PV} \int_{\mathbb{R}} \frac{F_{1}+F_{2}}{|x-y|^{1+2 p}} \chi_{E^{+}}(y) \mathrm{d} y<0 .
$$




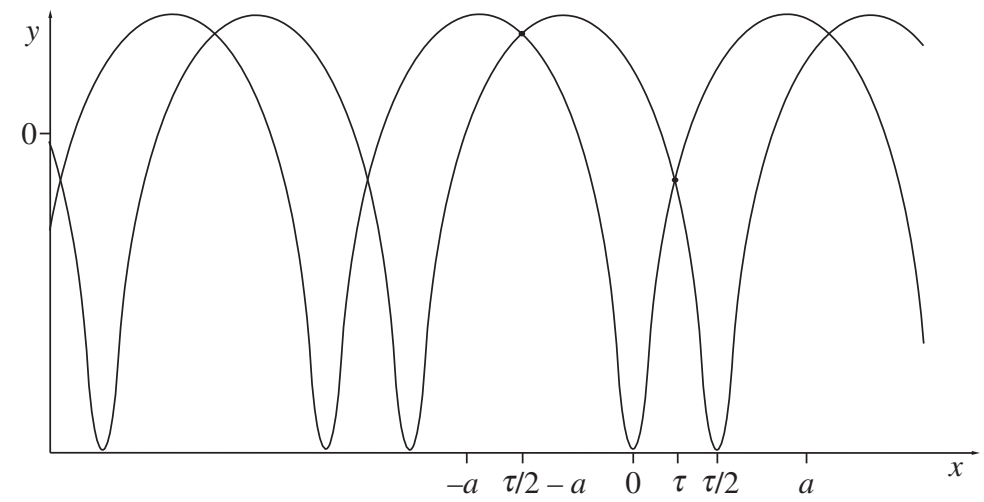

Figure 3. Intersection of periodic functions with special monotonicity properties.

Using the previous proposition, we can give the proof.

Proof of lemma 3.7. It is clear that $v^{\prime \prime}$ exists on $E^{+} \cup E^{-}$and is, due to proposition 3.8 , strictly positive on $E^{+}$and strictly negative on $E^{-}$. Furthermore, by the symmetry properties of $v$ one has $v^{\prime}(0)=0=v^{\prime}(a)$. We thus obtain for $x \in(0, a) \backslash\{\rho\}$,

$$
\begin{aligned}
& v^{\prime}(x)= \begin{cases}\int_{0}^{x}(-\Delta)^{1-s} g(y) \mathrm{d} y & \text { for } x \in(0, \rho), \\
-\int_{x}^{a}(-\Delta)^{1-s} g(y) \mathrm{d} y & \text { for } x \in(\rho, a),\end{cases} \\
& >0,
\end{aligned}
$$

where in the last step proposition 3.8 was used. Together with the continuity of $v$, the assertion follows.

The above monotonicity result yields certain elementary properties for the intersection points of shifted copies of the function $v$.

Proposition 3.9. For any translations $\tau_{1}, \tau_{2} \in \mathbb{R}$ and on any interval of the form $(\beta, \beta+2 a]$, the functions $v\left(\cdot-\tau_{1}\right)$ and $v\left(\cdot-\tau_{2}\right)$ either intersect each other exactly twice or they are identical, while in the first case the points of intersection have distance $a$.

Proof. Due to periodicity we can assume, without loss of generality, that $\tau_{1}=0$, $\beta=-a$ and $\tau_{2} \in(0, a]$. We denote by $w_{1}(\cdot):=v\left(\cdot-\tau_{1}\right)=v(\cdot), w_{2}:=v\left(\cdot-\tau_{2}\right)$ and $\tau:=\tau_{2}$. Assuming now that $\tau_{2}-\tau_{1}=\tau$ is not an integer multiple of $2 a$ and using the symmetry of $v$, we get

$$
w_{1}\left(\frac{1}{2} \tau\right)=v\left(\frac{1}{2} \tau\right)=v\left(-\frac{1}{2} \tau\right)=v\left(\frac{1}{2} \tau-\tau_{2}\right)=w_{2}\left(\frac{1}{2} \tau\right) .
$$




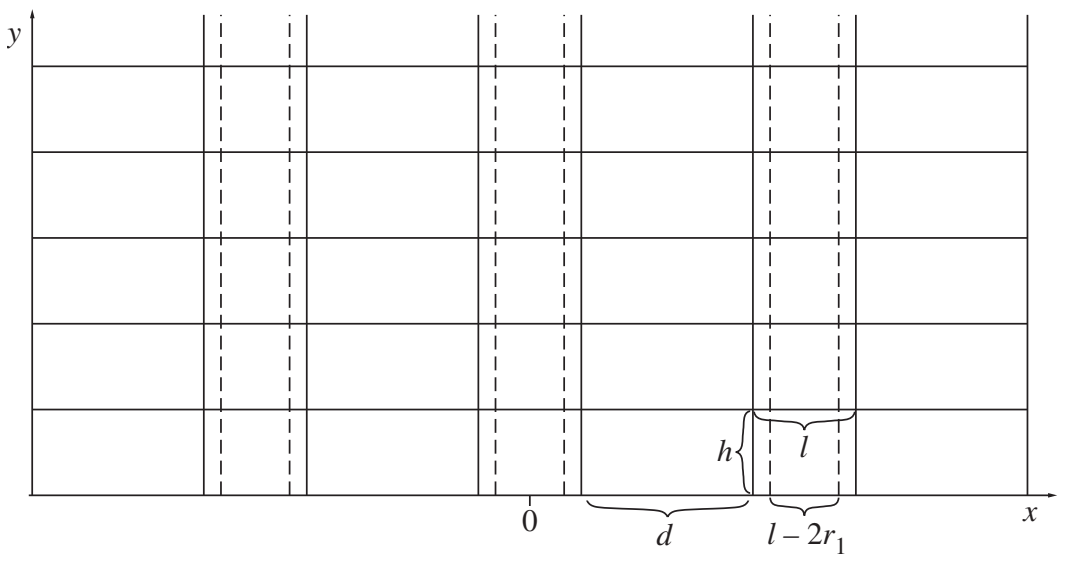

Figure 4 . Split-up of the plane $\mathbb{R} \times[0, \infty)$ into boxes.

Furthermore, using additionally the periodicity of $v$, we obtain

$$
\begin{aligned}
w_{1}\left(\frac{1}{2} \tau-a\right)=v\left(\frac{1}{2} \tau-a\right)=v\left(a-\frac{1}{2} \tau\right) & =v\left(-a-\frac{1}{2} \tau\right) \\
& =v\left(-a+\frac{1}{2} \tau-\tau\right) \\
& =w_{2}\left(\frac{1}{2} \tau-a\right) .
\end{aligned}
$$

Noting that $\tau / 2, \tau / 2-a \in(-a, a]$, we see that there are at least two points of intersection within $(-a, a]$ and they have distance $a$. Due to the monotonicity properties of $v$ there cannot be any other intersections in $(-a, a]$ (see figure 3 for an illustration).

Now we have collected all the properties of $v$ that we will need, so we continue constructing the stationary supersolution. Therefore, we split $\mathbb{R}^{2}$ into boxes with volume large enough that the probability of finding an obstacle centre in each box is larger than the critical probability $p_{H}(1)$ from theorem 2.1 . We can then apply the percolation result stated there.

Definition 3.10. For $k \in \mathbb{Z}, j \in \mathbb{N}$ and $l, d, h>0, l>2 r_{1}$, we define

$$
\begin{aligned}
Q_{k} & :=\left[k(l+d)-\frac{1}{2} l, k(l+d)+\frac{1}{2} l\right], \\
\tilde{Q}_{k} & :=\left[k(l+d)-\frac{1}{2} l+r_{1}, k(l+d)+\frac{1}{2} l-r_{1}\right], \\
\tilde{Q}_{k, j} & :=\tilde{Q}_{k} \times[(j-1) h, j h] .
\end{aligned}
$$

As a direct result of theorem 2.1 and assumption 1.1, we obtain the following proposition.

Proposition 3.11. Let $0<\alpha<1, H(k):=\left\lfloor k^{\alpha}\right\rfloor$ (i.e. the integer floor of $k^{\alpha}$ ) and let $V:=\left(l-2 r_{1}\right) h>0$ such that

$$
1-\exp \{-\lambda V\}>p_{H}(1)
$$


from theorem 2.1. ${ }^{1}$ Then, almost surely, there exist a function $\Lambda: \mathbb{Z} \rightarrow \mathbb{N}$ with $|\Lambda(x)-\Lambda(y)| \leqslant H(|x-y|)$ for all $x, y \in \mathbb{Z}$ and a mapping $I: \mathbb{Z} \rightarrow K$ with

$$
\left(x_{I(k)}, y_{I(k)}\right) \in \tilde{Q}_{k, \Lambda(k)}
$$

for all $k \in \mathbb{Z}$.

Note that for notational convenience we have dropped the dependence of $\Lambda$ on $\omega \in \Omega$. In the following we denote by $I$ the set $I(\mathbb{Z})$.

We now define a flat version of the aspired supersolution by projecting the obstacles, chosen by the proposition above, onto the real line and, locally around them, taking the minimum over translated versions of $v$. Explicitly, this is given by the following definition.

Definition 3.12. Now let $d \geqslant l, 2 a \geqslant d+2 l$ and define for $i \in I$,

$$
\begin{aligned}
u_{i}(x) & := \begin{cases}v\left(x-x_{i}\right) & \text { for } x \in\left[x_{i}-l-\frac{1}{2} d, x_{i}+l+\frac{1}{2} d\right], \\
+\infty & \text { otherwise, }\end{cases} \\
v_{i}(x) & :=v\left(x-x_{i}\right), \\
u_{\text {flat }}(x) & :=\min _{i \in I} u_{i}(x) .
\end{aligned}
$$

Proposition 3.13. With the definitions above, we have that:

(i) $u_{\text {flat }}$ is well defined, bounded and continuous;

(ii) given $i \in I$ and $k \in \mathbb{N}$ such that $x_{i} \in \tilde{Q}_{k}$, we have $u_{\text {flat }}(x)=v_{i}(x)$ for all $x \in Q_{k}$.

Proof. (i) For every $x \in \mathbb{R}$ there are only finitely many $u_{i}$ having a finite value in $x$. Furthermore, for $k \in \mathbb{N}$ and $i, j \in I$ with $x_{i} \in \tilde{Q}_{k}$ and $x_{j} \in \tilde{Q}_{k+1}$, due to proposition 3.9, the functions $u_{i}$ and $u_{j}$ intersect and are both finite in $\left(x_{i}+x_{j}\right) / 2$. Thus, $u_{\text {flat }}$ is well defined as well as bounded and continuous, as $v$ is bounded and continuous.

(ii) It suffices to show that two functions $u_{i} \not \equiv u_{j}, i, j \in I$, have no intersection (with the exception of $\infty$ ) inside $Q_{k}$. For $x_{i} \in Q_{m}$ and $x_{j} \in Q_{n}$ with $|m-n| \geqslant 2$ this is clear by the definitions of $u_{i}$ and $u_{j}$. Let $x_{i} \in Q_{k}$ and $x_{j} \in Q_{k+1}$. It is then enough to show that $u_{i}$ and $u_{j}$ intersect neither inside $Q_{k}$ nor inside $Q_{k+1}$. From the periodicity one knows that $u_{i}$ and $u_{j}$ intersect in $x_{i}+\left|x_{j}-x_{i}\right| / 2=x_{j}-\left|x_{j}-x_{i}\right| / 2=$ $\left(x_{i}+x_{j}\right) / 2$, where $x_{j}>x_{i}$, due to the fact that $x_{i} \in Q_{k}, x_{j} \in Q_{k+1}$. This property together with the definition of $Q_{k}$ and $Q_{k+1}$ also gives

$$
\begin{gathered}
k(l+d)-\frac{1}{2} l+r_{1} \leqslant x_{i} \leqslant k(l+d)+\frac{1}{2} l-r_{1} \\
(k+1)(l+d)-\frac{1}{2} l+r_{1} \leqslant x_{j} \leqslant(k+1)(l+d)+\frac{1}{2} l-r_{1} .
\end{gathered}
$$

${ }^{1}$ We take $p_{H}(1)$ to be the value from the proof of the theorem in order to be able to apply remark 2.3 . 
Adding the two inequalities and dividing by 2 gives

$$
\begin{aligned}
K(l+d)+\frac{l+d}{2}-\frac{l}{2}+r_{1} & \leqslant \frac{x_{i}+x_{j}}{2} \\
& \leqslant k(l+d)+\frac{l+d}{2}+\frac{l}{2}-r_{1} \\
& =(k+1)(l+d)-\frac{l}{2}+\frac{l}{2}-\frac{d}{2}-r_{1} .
\end{aligned}
$$

Now using that $r_{1}>0$ and $l \leqslant d$, one gets

$$
\begin{aligned}
k(l+d)+\frac{l}{2} & <k(l+d)+\frac{l+d}{2}-\frac{l}{2}+r_{1} \\
& \leqslant \frac{x_{i}+x_{j}}{2} \\
& \leqslant(k+1)(l+d)-\frac{l}{2}-\frac{d-l}{2}-r_{1} \\
& <(k+1)(l+d)-\frac{l}{2} .
\end{aligned}
$$

Then $\left(x_{i}+x_{j}\right) / 2 \notin Q_{k}, Q_{k+1}$ by the definition of $Q_{k}$ and $Q_{k+1}$. As the next point of intersection has distance $a$ to $\left(x_{i}+x_{j}\right) / 2$ and $a \geqslant d+l / 2$ this shows the claim.

We next prove that $u_{\text {flat }}$ is a supersolution to a modified equation where the obstacles are projected to the real line, meaning that for the moment we neglect the $y$ direction. This will be done by estimating the effect of the fractional Laplacian for any fixed $\xi \in \mathbb{R}$. In order to perform such an estimate, we first need to fix some notation.

Definition 3.14. Let $\xi \in \mathbb{R}$ and $i_{0} \in I$ be such that $u_{\text {flat }}(\xi)=u_{i_{0}}(\xi)=v_{i_{0}}(\xi)$. If $u_{i}(\xi)=u_{j}(\xi)=u_{\text {flat }}(\xi)$, we take $i_{0}=\max \{i, j\}$. Furthermore, we recursively define the points of (proper) intersection of the periodic function $v_{i_{0}}$ with $u_{\text {flat }}$. Let

$$
\begin{aligned}
& a_{1}:=\min \left\{y \geqslant 0: \exists \kappa>0 \text { with } \begin{array}{l}
v_{i_{0}}>u_{\text {flat }} \text { on }(\xi-y-\kappa, \xi-y) \\
v_{i_{0}} \leqslant u_{\text {flat }} \text { on }(\xi-y, \xi-y+\kappa)
\end{array}\right\}, \\
& b_{1}:=\min \left\{y \geqslant a_{1}: \exists \kappa>0 \text { with } \begin{array}{l}
v_{i_{0}}<u_{\text {flat }} \text { on }(\xi-y-\kappa, \xi-y) \\
v_{i_{0}}>u_{\text {flat }} \text { on }(\xi-y, \xi-y+\kappa)
\end{array}\right\}, \\
& a_{k+1}:=\min \left\{y \geqslant b_{k}: \exists \kappa>0 \text { with } \begin{array}{l}
v_{i_{0}}>u_{\text {flat }} \text { on }(\xi-y-\kappa, \xi-y) \\
v_{i_{0}}<u_{\text {flat }} \text { on }(\xi-y, \xi-y+\kappa)
\end{array}\right\}, \\
& b_{k+1}:=\min \left\{y \geqslant a_{k+1}: \exists \kappa>0 \text { with } \begin{array}{l}
v_{i_{0}}<u_{\text {flat }} \text { on }(\xi-y-\kappa, \xi-y) \\
v_{i_{0}}>u_{\text {flat }} \text { on }(\xi-y, \xi-y+\kappa)
\end{array}\right\} .
\end{aligned}
$$

Define $\tilde{a}_{k}$ and $\tilde{b}_{k}$ in the same way by substituting $+y$ for $-y$.

With this definition we can state the main ingredient for the following estimates.

LEMMA 3.15. We have

$$
b_{k}-a_{k} \geqslant a \text { and } a_{k+1}-b_{k} \leqslant a
$$

for all $k \in \mathbb{N}$. 


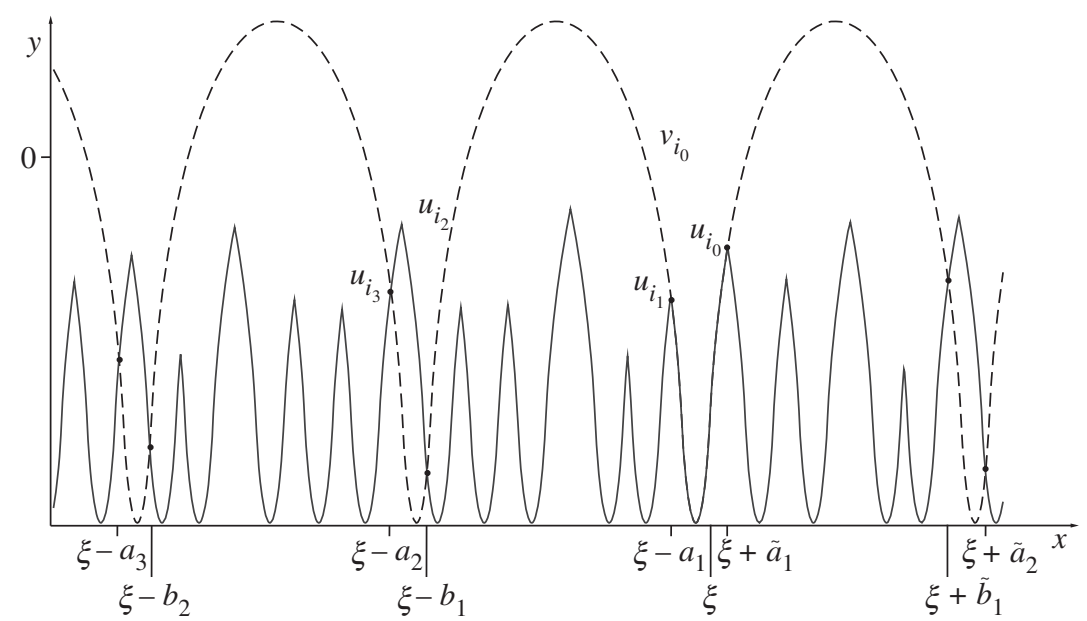

Figure 5. Illustration for the definition of and distance between intersection points of local supersolutions with the periodic supersolution $v_{i_{0}}$.

Proof. Take $k \in \mathbb{N}$ arbitrarily and $i_{1}, i_{2} \in I$ such that $u_{i_{1}}\left(\xi-a_{k}\right)=u_{\text {flat }}\left(\xi-a_{k}\right)$ and $u_{i_{2}}\left(\xi-b_{k}\right)=u_{\text {flat }}\left(\xi-b_{k}\right)$ (it is clear by construction that $i_{1}$ and $i_{2}$ are unique).

Because of proposition 3.9 and the construction of $u_{\mathrm{flat}}$, one has

$$
u_{\text {flat }} \leqslant v_{i_{1}} \quad \text { on }\left[\xi-a_{k}-a, \xi-a_{k}\right] .
$$

The definition of $a_{k}$ and proposition 3.9 yield

$$
v_{i_{0}}\left(\xi-a_{k}\right)=v_{i_{1}}\left(\xi-a_{k}\right) \text { and } v_{i_{0}}(x)>v_{i_{1}}(x) \text { for } x \in\left(\xi-a_{k}-a, \xi-a_{k}\right) .
$$

Altogether one has

$$
v_{i_{0}}(x)>v_{i_{1}}(x) \geqslant u_{\text {flat }}(x) \text { for all } x \in\left(\xi-a_{k}-a, \xi-a_{k}\right) .
$$

Furthermore,

$$
u_{\text {flat }} \leqslant v_{i_{0}} \text { on }\left[\xi-b_{k}, \xi-a_{k}\right]
$$

and by the choice of $a_{k}$ and $b_{k}$ there exists no larger interval $J \supseteq\left[\xi-b_{k}, \xi-a_{k}\right]$ with $u_{\text {flat }} \leqslant v_{i_{0}}$ on $J$. Using this, one obtains

$$
\left(\xi-a_{k}-a, \xi-a_{k}\right) \subset\left[\xi-b_{k}, \xi-a_{k}\right]
$$

and therefore

$$
b_{k}-a_{k} \geqslant a \text {. }
$$

The other inequality is shown by an explicit calculation. We define $r:=2\left(x_{i_{2}}-\right.$ $\left.\left(\xi-b_{k}\right)\right)$ and $z_{0}:=x_{i_{2}}-r$. Then, by the periodicity property of $v_{i_{0}}$ and $v_{i_{2}}$, it is clear that $z_{0}$ is a minimum of $v_{i_{0}}$. Furthermore, let $i_{3} \in I$ be such that $x_{i_{3}}$ is the next minimum of $u_{\text {flat }}$ to the left of $x_{i_{2}}$. By construction of $u_{\text {flat }}$, it is obvious that one has $u_{i_{3}}\left(\xi-a_{k+1}\right)=u_{\text {flat }}\left(\xi-a_{k+1}\right)$. Furthermore, one has $x_{i_{3}}<z_{0}<x_{i_{2}}$. From the proof of proposition 3.13 one then knows that $v_{i_{0}}$ and $u_{i_{3}}$ intersect in

$$
\xi-a_{k+1}=\frac{x_{i_{3}}+z_{0}}{2} .
$$




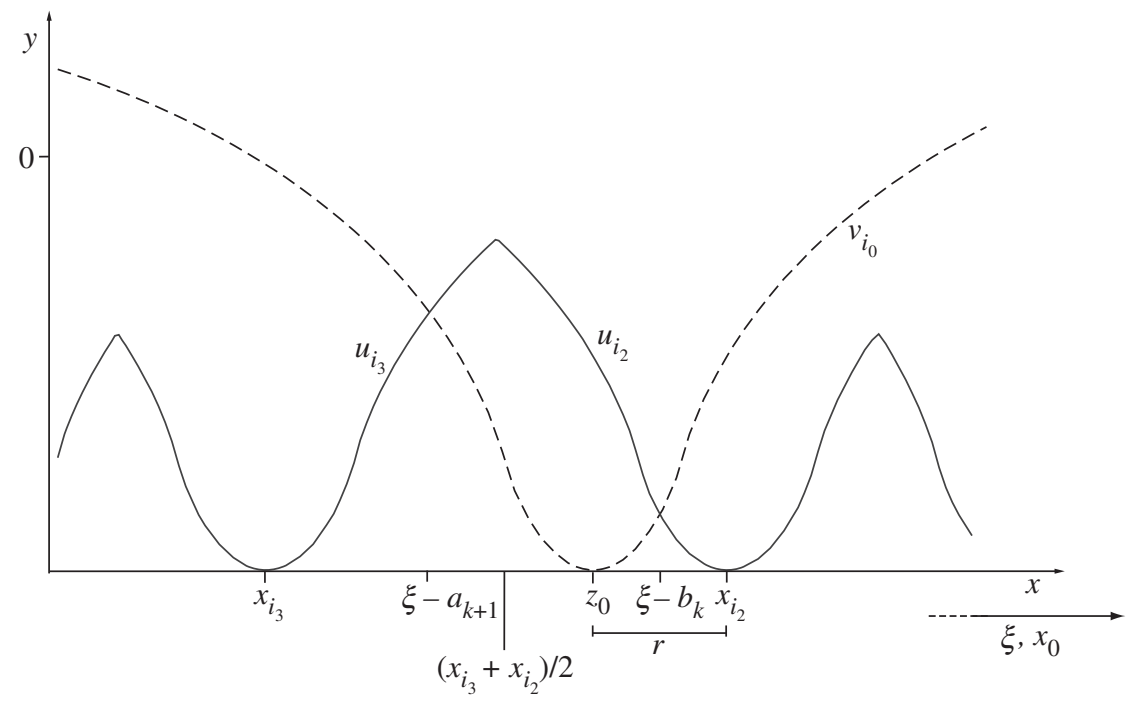

Figure 6. Illustration to estimate the distance between intersection points of local supersolutions and the periodic supersolution $v_{i_{0}}$.

The same argument also shows for the intersection of $v_{i_{0}}$ and $u_{i_{2}}$,

$$
\xi-b_{k}=\frac{x_{i_{2}}+z_{0}}{2} .
$$

By the choice of the $Q_{n}$, it follows that $\left|x_{i_{2}}-x_{i_{3}}\right|=x_{i_{2}}-x_{i_{3}} \leqslant d+2 l-2 r_{1}$ and therefore $\left|x_{i_{2}}-x_{i_{3}}\right| / 2 \leqslant d / 2+l-r_{1}<a$. Putting everything together, one gets

$$
a_{k+1}-b_{k}=\left(\xi-b_{k}\right)-\left(\xi-a_{k+1}\right)=\frac{x_{i_{2}}+z_{0}}{2}-\frac{x_{i_{3}}+z_{0}}{2}=\frac{x_{i_{2}}-x_{i_{3}}}{2}<a .
$$

See figure 6 for an illustration.

REMARK 3.16. In the same way it follows that

$$
\tilde{b}_{k}-\tilde{a}_{k} \geqslant a \text { and } \tilde{a}_{k+1}-\tilde{b}_{k} \leqslant a .
$$

For the case in which $\xi$ is a point where $u_{\text {flat }}$ is smooth, there is no issue applying the integral representation directly in order to show that $u_{\text {flat }}$ is a supersolution to our modified problem. However, at points where $u_{\text {flat }}$ is only continuous, in particular, where the representing $u_{i}$ changes or where $g$ admits a jump, we have to be slightly more careful (at least for the case in which $s \geqslant 1 / 2$ ).

Definition 3.17. We define the set $D$ of points where $u_{\text {flat }}$ is not smooth (i.e. $C^{2}$ ) consisting of the set

$$
D_{1}:=\left\{z_{k} \pm \rho: k \in \mathbb{Z}\right\}
$$

of points where the (shifted copy of the) function $g$ admits a jump and the set

$$
D_{2}:=\left\{\frac{z_{k}+z_{k+1}}{2}: k \in \mathbb{Z}\right\}
$$


of points where the functions $v_{i}$ representing $u_{\text {flat }}$ change. We use here the following notation: for every $k \in \mathbb{Z}$ we define $z_{k}$ to be the unique point $x_{i} \in \tilde{Q}_{k}$ with $i \in I$. We then have $D=D_{1} \cup D_{2}$.

For $\xi \notin D$ we now can prove the following estimate.

Lemma 3.18. For $\xi \notin D$ (for $s<1 / 2$ we only need $\xi \notin D_{1}$ ) we have

$$
\mathrm{PV} \int_{\mathbb{R}} \frac{u_{\mathrm{flat}}(y)-u_{\mathrm{flat}}(\xi)}{|y-\xi|^{1+2 s}} \mathrm{~d} y \leqslant \mathrm{PV} \int_{\mathbb{R}} \frac{v_{i_{0}}(y)-v_{i_{0}}(\xi)}{|y-\xi|^{1+2 s}} \mathrm{~d} y .
$$

REMARK 3.19. The idea behind the following proof is that we compare the area between the graphs of $u_{\text {flat }}$ and $v_{i_{0}}$, while this area is weighted by the kernel of the integral representation of $(-\Delta)^{s}$. In this situation the intervals where $u_{\text {flat }}<v_{i_{0}}$ give some negative contribution, while the intervals with $u_{\text {flat }}>v_{i_{0}}$ give some positive contribution. Using lemma 3.15 it is possible to show that the total sum of all these 'weighted signed areas' is negative.

Proof of lemma 3.18. The explicit calculation is done only for the part to the left of $\xi$. The estimate for the other part follows analogously. Using the same notation as in lemma 3.15 , one has $u_{\text {flat }} \leqslant v_{i_{0}}$ on $\left[\xi-b_{k}, \xi-a_{k}\right]$, and so the definition of $u_{\text {flat }}$ together with lemma 3.15 yields

$$
\begin{aligned}
\int_{\xi-b_{k}}^{\xi-a_{k}} u_{\text {flat }}(y) & -v_{i_{0}}(y) \mathrm{d} y \\
& \leqslant \min \left\{\int_{\xi-b_{k}}^{\xi-b_{k}+a} v_{i_{2}}(y)-v_{i_{0}}(y) \mathrm{d} y, \int_{\xi-a_{k}-a}^{\xi-a_{k}} v_{i_{1}}(y)-v_{i_{0}}(y) \mathrm{d} y\right\} \\
& \leqslant \int_{\xi-b_{k}}^{\xi-b_{k}+a} v_{i_{2}}(y)-v_{i_{0}}(y) \mathrm{d} y \\
& \leqslant 0
\end{aligned}
$$

In the same way, using $v_{i_{0}} \leqslant u_{\text {flat }}$ on $\left[\xi-a_{k+1}, \xi-b_{k}\right]$, we get

$$
\begin{aligned}
0 & \leqslant \int_{\xi-a_{k+1}}^{\xi-b_{k}} u_{\text {flat }}(y)-v_{i_{0}}(y) \mathrm{d} y \\
& \leqslant \min \left\{\int_{\xi-a_{k+1}}^{\xi-a_{k+1}+a} v_{i_{3}}(y)-v_{i_{0}}(y) \mathrm{d} y, \int_{\xi-b_{k}-a}^{\xi-b_{k}} v_{i_{2}}(y)-v_{i_{0}}(y) \mathrm{d} y\right\} \\
& \leqslant \int_{\xi-b_{k}-a}^{\xi-b_{k}} v_{i_{2}}(y)-v_{i_{0}}(y) \mathrm{d} y .
\end{aligned}
$$

Using this, we can split up

$$
\int_{\xi-a_{k+1}}^{\xi-a_{k}} \frac{u_{\mathrm{flat}}(y)-v_{i_{0}}(y)}{|y-\xi|^{1+2 s}} \mathrm{~d} y
$$

into the two parts where the integrand is negative and positive, respectively (see figure 7 for an illustration). Noticing that $1 /|y-\xi|^{1+2 s} \leqslant 1 / b_{k}^{1+2 s}$ on $\left[\xi-a_{k+1}, \xi-b_{k}\right]$ 
and $1 / b_{k}^{1+2 s} \leqslant 1 /|y-\xi|^{1+2 s}$ on $\left[\xi-b_{k}, \xi-a_{k}\right]$, we obtain

$$
\begin{aligned}
& \int_{\xi-a_{k+1}}^{\xi-a_{k}} \frac{u_{\mathrm{flat}}(y)-v_{i_{0}}(y)}{|y-\xi|^{1+2 s}} \mathrm{~d} y \\
& \quad \leqslant \frac{1}{b_{k}^{1+2 s}} \int_{\xi-a_{k+1}}^{\xi-b_{k}} u_{\mathrm{flat}}(y)-v_{i_{0}}(y) \mathrm{d} y+\frac{1}{b_{k}^{1+2 s}} \int_{\xi-b_{k}}^{\xi-a_{k}} u_{\mathrm{flat}}(y)-v_{i_{0}}(y) \mathrm{d} y \\
& \quad \leqslant \frac{1}{b_{k}^{1+2 s}} \int_{\xi-b_{k}-a}^{\xi-b_{k}+a} v_{i_{2}}(y)-v_{i_{0}}(y) \mathrm{d} y \\
& =0
\end{aligned}
$$

where in the last step we used that the average of $v_{i_{0}}$ and $v_{i_{2}}$ vanishes. Inserting a zero of the form $-v_{i_{0}}(\xi)+v_{i_{0}}(\xi)$, we obtain

$$
0 \geqslant \int_{\xi-a_{k+1}}^{\xi-a_{k}} \frac{u_{\text {flat }}(y)-v_{i_{0}}(\xi)}{|y-\xi|^{1+2 s}} \mathrm{~d} y-\int_{\xi-a_{k+1}}^{\xi-a_{k}} \frac{v_{i_{0}}(y)-v_{i_{0}}(\xi)}{|y-\xi|^{1+2 s}} \mathrm{~d} y .
$$

Now using $v_{i_{0}}(\xi)=u_{\text {flat }}(\xi)$ and summing up for all $k$ in $\mathbb{N}$, it follows that

$$
\int_{-\infty}^{\xi-a_{1}} \frac{u_{\text {flat }}(y)-u_{\text {flat }}(\xi)}{|y-\xi|^{1+2 s}} \mathrm{~d} y \leqslant \int_{-\infty}^{\xi-a_{1}} \frac{v_{i_{0}}(y)-v_{i_{0}}(\xi)}{|y-\xi|^{1+2 s}} \mathrm{~d} y .
$$

Furthermore, an analogous calculation shows that

$$
\int_{\xi+\tilde{a}_{1}}^{\infty} \frac{u_{\mathrm{flat}}(y)-u_{\mathrm{flat}}(\xi)}{|y-\xi|^{1+2 s}} \mathrm{~d} y \leqslant \int_{\xi+\tilde{a}_{1}}^{\infty} \frac{v_{i_{0}}(y)-v_{i_{0}}(\xi)}{|y-\xi|^{1+2 s}} \mathrm{~d} y,
$$

which, together with $u_{\text {flat }}=v_{i_{0}}$ on $\left(\xi-a_{1}, \xi+\tilde{a}_{1}\right)$, yields

$$
\mathrm{PV} \int_{\mathbb{R}} \frac{u_{\text {flat }}(y)-u_{\text {flat }}(\xi)}{|y-\xi|^{1+2 s}} \mathrm{~d} y \leqslant \mathrm{PV} \int_{\mathbb{R}} \frac{v_{i_{0}}(y)-v_{i_{0}}(\xi)}{|y-\xi|^{1+2 s}} \mathrm{~d} y .
$$

Definition 3.20. Let $\rho<r_{0}$ and $F_{2}<q$. We then define

$$
g_{\text {flat }}(x):= \begin{cases}q & \text { for } x \in \bigcup_{i \in I}\left[x_{i}-r_{0}, x_{i}+r_{0}\right], \\ 0 & \text { for } x \notin \bigcup_{i \in I}\left[x_{i}-r_{0}, x_{i}+r_{0}\right] .\end{cases}
$$

Proposition 3.21. Under the conditions from definition 3.20 we have

$$
\mathrm{A} u_{\text {flat }}-g_{\text {flat }}+F \leqslant 0
$$

for every $F \leqslant \min \left\{q-F_{2}, F_{1}\right\}$.

Proof. For $x \notin D$ this is already clear from lemma 3.18 and the definition of $g_{\text {flat }}$. Furthermore, for $x \in D_{1}$ we know from the construction of $v$ that $\lim _{y \rightarrow x_{-}} \mathrm{A} u_{\text {flat }}(y)$ and $\lim _{y \rightarrow x_{+}} \mathrm{A} u_{\text {flat }}(y)$ exist and from lemma 3.18 it is clear that

$$
\lim _{y \rightarrow x_{ \pm}} \mathrm{A} u_{\text {flat }}(y) \leqslant \lim _{y \rightarrow x_{ \pm}} \mathrm{A} v_{i(x)}(y) .
$$

From the construction of $g_{\text {flat }}$ we see that $\lim _{y \rightarrow x_{ \pm}} \mathrm{A} v_{i(x)}(y)+F \leqslant g_{\text {flat }}(x)$. 


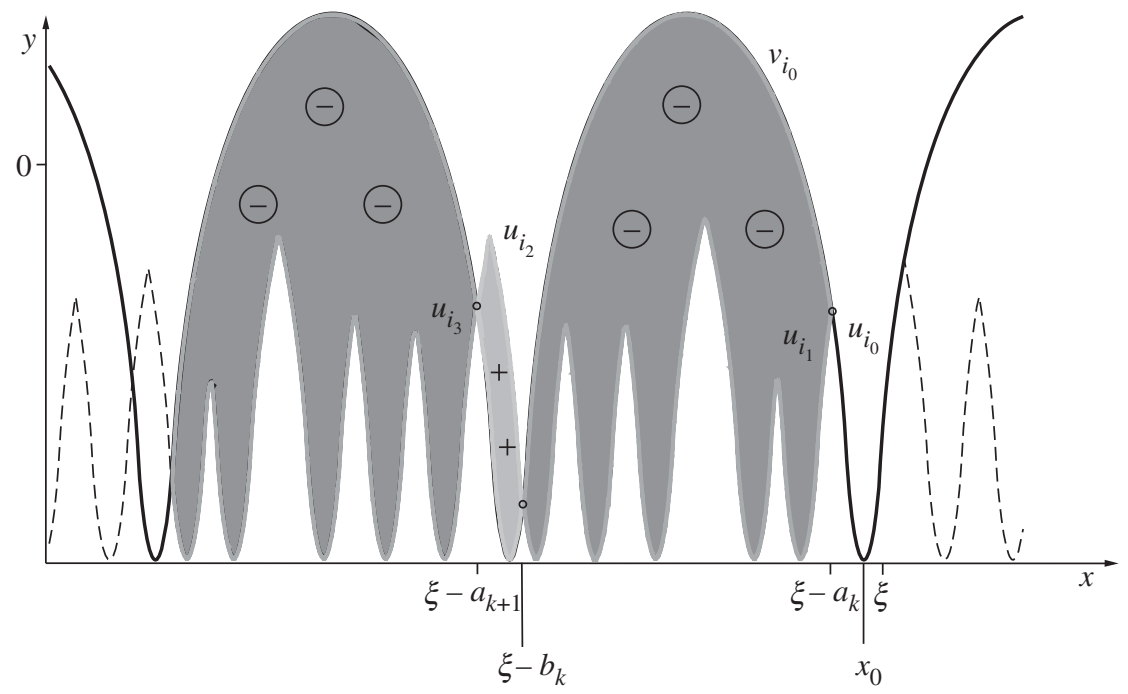

Figure 7. Illustration to estimate and compare the area between the graphs of $u_{\text {flat }}$ and $v_{i_{0}}$.

For $s<1 / 2$ the case $x \in D_{2}$ is already proven in lemma 3.18. Let $s \geqslant 1 / 2$ and $x \in D_{2}$. Since the derivative of $u_{\text {flat }}$ makes a downward jump at $x$, we have that A $u_{\text {flat }}(x)=-\infty$ and therefore we also have $\mathrm{A} u_{\text {flat }}(x)+F \leqslant g_{\text {flat }}(x)$.

We are now prepared to perform the last step in constructing our supersolution $u$. That is, we will lift $u_{\text {smooth }}$ locally to the obstacles chosen for the percolation cluster. Technically this will be done by just adding a smoothed piecewise constant function for which we first collect some properties.

Proposition 3.22. Let $h>0, d>0, l>0, s \in(0,1)$. For $\Lambda: \mathbb{Z} \rightarrow \mathbb{R}$ such that $\left|\Lambda\left(z_{1}\right)-\Lambda\left(z_{2}\right)\right| \leqslant 2 h\left|z_{1}-z_{2}\right|^{\alpha}$ with $0<\alpha<2 s$ there exist a smooth function $u_{\text {lift }}: \mathbb{R} \rightarrow \mathbb{R}$ and constants $C_{0}, C_{1}$ and $C_{2}$ that only depend on $s$ and $\alpha$ such that:

(i) $u_{\text {lift }}(x)=\Lambda(k)$ for any $x \in Q_{k}, k \in \mathbb{Z}$;

(ii) $\left\|\partial_{x}^{2} u_{\text {lift }}\right\|_{L^{\infty}} \leqslant C_{0} \frac{h}{d^{2}}$;

(iii) $\left|(-\Delta)^{s} u_{\text {lift }}(x)\right| \leqslant C_{1} \frac{(d / 2+l / 2)^{2-2 s}}{d^{2}} h+C_{2} \frac{h}{(d / 2+l / 2)^{2 s}}$.

Proof. Parts (i) and (ii) follow immediately if we define $u_{\text {lift }}:=\tilde{u}_{\text {lift }} * \eta_{d / 2}$, where $\tilde{u}_{\text {lift }}$ is defined as the piecewise constant function

$$
\tilde{u}_{\text {lift }}(x):=\Lambda(k) \quad \text { for } x \in\left(k(l+d)-\frac{1}{2} l-\frac{1}{2} d, k(l+d)+\frac{1}{2} l+\frac{1}{2} d\right] .
$$

To see part (iii), we assume without loss of generality that

$$
x \in\left[-\frac{1}{2} d-\frac{1}{2} l, \frac{1}{2} d+\frac{1}{2} l\right] .
$$


Let $\Pi:=(-3(d / 2+l / 2), 3(d / 2+l / 2))$. We then have, from the assumptions on $\Lambda$ and from (i), for all $y \in \mathbb{R} \backslash \Pi$,

$$
\left|u_{\text {lift }}(x)-u_{\text {lift }}(y)\right| \leqslant 6 h \frac{|x-y|^{\alpha}}{(l+d)^{\alpha}}
$$

because

$$
\left|u_{\text {lift }}(x)-u_{\text {lift }}(y)\right| \leqslant 2 h \frac{|x-y|^{\alpha}}{(l+d)^{\alpha}}+4 h
$$

and for $y \in \mathbb{R} \backslash \Pi$ one has $|x-y|^{\alpha} /(l+d)^{\alpha} \geqslant 1$ and therefore $4 h \leqslant 4 h|x-y|^{\alpha} /(l+d)^{\alpha}$.

Using that $u_{\text {lift }}$ is smooth and grows less than linearly, $(-\Delta)^{s} u_{\text {lift }}$ can be represented by its integral form as

$$
(-\Delta)^{s} u_{\text {lift }}(x)=-\frac{C}{2} \int_{\mathbb{R}} \frac{u_{\text {lift }}(x-y)+u_{\text {lift }}(x+y)-2 u_{\text {lift }}(x)}{|y|^{1+2 s}} \mathrm{~d} y .
$$

By applying some standard estimates [3] we get

$$
\begin{aligned}
\left|(-\Delta)^{s} u_{\text {lift }}(x)\right|= & \frac{C}{2}\left|\int_{\mathbb{R}} \frac{u_{\text {lift }}(x-y)+u_{\text {lift }}(x+y)-2 u_{\text {lift }}(x)}{|y|^{1+2 s}} \mathrm{~d} y\right| \\
\leqslant & C \frac{\left\|\partial_{x}^{2} u_{\text {lift }}\right\|_{L^{\infty}}}{2} \int_{\Pi} \frac{1}{|y|^{2 s-1}} \mathrm{~d} y \\
& +\frac{C}{2} \int_{\mathbb{R} \backslash \Pi} \frac{\left|u_{\text {lift }}(x-y)-u_{\text {lift }}(x)\right|+\left|u_{\text {lift }}(x+y)-u_{\text {lift }}(x)\right|}{|y|^{1+2 s}} \mathrm{~d} y .
\end{aligned}
$$

Putting in (3.1) for $u_{\text {lift }}$ and calculating the resulting integrals, it follows that

$$
\begin{aligned}
& \left|(-\Delta)^{s} u_{\text {lift }}(x)\right| \\
& \leqslant C\left\|\partial_{x}^{2} u_{\text {lift }}\right\|_{L^{\infty}} \int_{0}^{3(d / 2+l / 2)} \frac{1}{y^{2 s-1}} \mathrm{~d} y+C \frac{6 h}{(l+d)^{\alpha}} \int_{\mathbb{R} \backslash \Pi} \frac{1}{|y|^{1+2 s-\alpha}} \mathrm{d} y \\
& \quad \leqslant C \frac{\left\|\partial_{x}^{2} u_{\text {lift }}\right\|_{L^{\infty}}}{2-2 s} 3^{2-2 s}\left(\frac{d}{2}+\frac{l}{2}\right)^{2-2 s}+C \frac{12 h}{(l+d)^{\alpha}} \int_{3(d / 2+l / 2)}^{\infty} y^{-1-2 s+\alpha} \mathrm{d} y \\
& \quad \leqslant C \frac{\left\|\partial_{x}^{2} u_{\text {lift }}\right\|_{L^{\infty}}}{2-2 s} 3^{2-2 s}\left(\frac{d}{2}+\frac{l}{2}\right)^{2-2 s}+C \frac{12 h}{2 s-\alpha} 3^{\alpha-2 s} \frac{(d / 2+l / 2)^{-2 s+\alpha}}{(d+l)^{\alpha}} \\
& \quad \leqslant C \frac{3^{2-2 s}}{2-2 s} C_{0} \frac{h}{d^{2}}\left(\frac{d}{2}+\frac{l}{2}\right)^{2-2 s}+C \frac{12}{2 s-\alpha} \frac{3^{\alpha-2 s}}{2^{\alpha}} h\left(\frac{d}{2}+\frac{l}{2}\right)^{-2 s},
\end{aligned}
$$

where in the last step $\left\|\partial_{x}^{2} u_{\text {lift }}\right\|_{L^{\infty}} \leqslant C_{0} h / d^{2}$ was used. For

$$
C_{1}=C \frac{3^{2-2 s}}{2-2 s} C_{0} \quad \text { and } \quad C_{2}=C \frac{12}{2 s-\alpha} \frac{3^{\alpha-2 s}}{2^{\alpha}}
$$

we obtain the estimate.

REMARK 3.23. Note that in item (iii) the restriction $\alpha<2 s$ for the case in which $s>1 / 2$ gives $\alpha \leqslant 1$, and thus it suffices if $\Lambda$ is Lipschitz continuous and therefore 


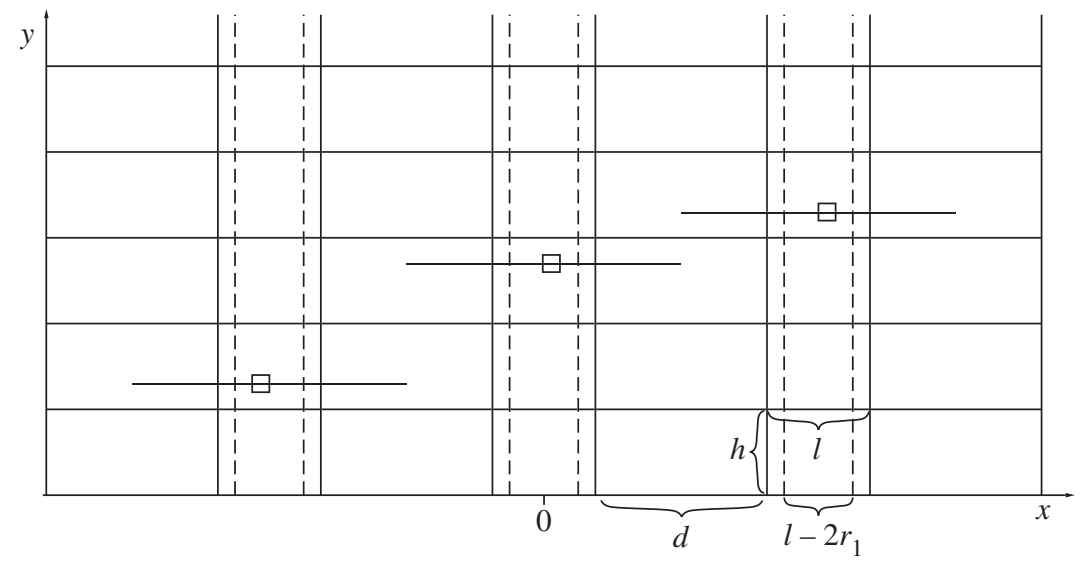

Figure 8. Illustration for the construction of $u_{\text {lift }}$; the first step.

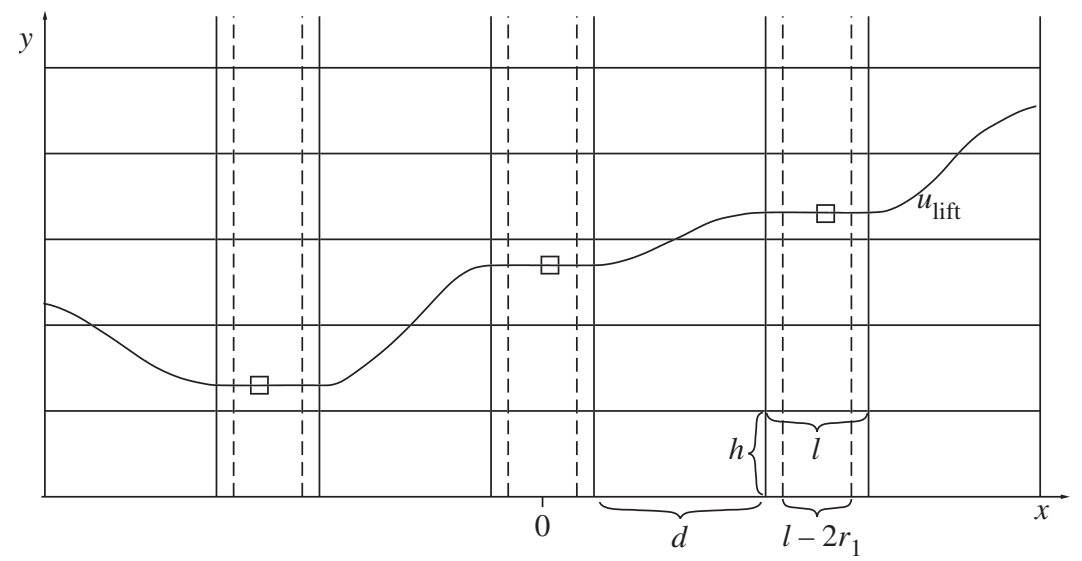

Figure 9. Illustration for the construction of $u_{\text {lift }}$; the second step.

we could use Lipschitz percolation from [4]. But for $s \leqslant 1 / 2$, in order to make the integral finite, the stronger percolation result in theorem 2.1 is necessary.

We next summarize the scaling properties that we will need to finally prove our main theorem for the case in which $1 / 2<s<1$.

Lemma 3.24. Let $1 / 2<s<1$ and take $C_{\infty}:=\left(2 / \pi^{2 s}\right) \zeta(2 s)$ and $C_{a}>5$.

Take $q>0$ and $V>0$ as in proposition 3.11. Choose $0<F_{2}<q$ and take $F_{1}>0$ as in definition 3.1. Now choose $l>0$ such that

$$
\begin{aligned}
l>\max \left\{4 r_{1},\left(\frac{\left(C_{1}+C_{2}\right) V}{r_{1}\left(q-F_{2}\right)}\right)^{1 /(2 s)},\left(12\left(C_{1}+C_{2}\right) V r_{0}\right)^{1 /(2 s)},\right. \\
\left.\frac{1}{\left(C_{\infty}\left(\frac{3}{2}\right)^{2 s-1} F_{2}\right)^{1 /(2 s-1)}}, \frac{1+2 F_{2} r_{0} r_{1}+12 F_{2}\left(C_{1}+C_{2}\right) V C_{\infty} C_{a}^{2 s}}{F_{2} r_{0}}\right\} .
\end{aligned}
$$


Take $d=l, 3 l / 2=d / 2+l \leqslant a \leqslant C_{a} l, \rho=a r_{0} / 3\left(C_{\infty} F_{2} a^{2 s}+r_{0}\right)$ and $h=V /\left(l-2 r_{1}\right)$. Then we have:

(i) $\rho<\frac{r_{0}}{3}<\frac{a}{18}$,

(ii) $\left(C_{1}+C_{2}\right) V \frac{1}{l^{2 s}} \frac{1}{l-2 r_{1}}<\frac{q-F_{2}}{2}$,

(iii) $\left(C_{1}+C_{2}\right) V \frac{1}{l^{2 s}} \frac{1}{l-2 r_{1}}<\frac{1}{12} \frac{r_{0}}{\left(C_{\infty} C_{a}^{2 s} F_{2} l^{2 s}+r_{0}\right)}$,

(iv) $\|v\|_{L^{\infty}}<\frac{r_{0}}{2}$

(v) $-F_{1} \leqslant-\frac{1}{6} \frac{r_{0} F_{2}}{\left(C_{\infty} C_{a}^{2 s} F_{2} l^{2 s}+r_{0}\right)}$.

Proof. (i) The choice of $b, \delta, a$ and $l$ yields

$$
\begin{aligned}
\rho=\frac{1}{3} \frac{a r_{0}}{C_{\infty} F_{2} a^{2 s}+r_{0}}<\frac{1}{3} \frac{r_{0}}{C_{\infty} F_{2} a^{2 s-1}} & \leqslant \frac{1}{3} \frac{r_{0}}{C_{\infty} F_{2}\left(\frac{3}{2}\right)^{2 s-1} l^{2 s-1}} \\
& <\frac{r_{0}}{3} \frac{1}{C_{\infty} F_{2}\left(\frac{3}{2}\right)^{2 s-1}\left(1 / C_{\infty} F_{2}\left(\frac{3}{2}\right)^{2 s-1}\right)} \\
& =\frac{r_{0}}{3} .
\end{aligned}
$$

From the conditions on $r_{0}, r_{1}$ and the choice of $l$, one also gets

$$
\frac{r_{0}}{3}<\frac{r_{1}}{3}<\frac{l}{12} \leqslant \frac{2}{3} \frac{a}{12}=\frac{a}{18} .
$$

(ii) From the conditions on $l$ we get

$$
\begin{aligned}
l & >\left(\frac{2\left(C_{1}+C_{2}\right) V}{2 r_{1}\left(q-F_{2}\right)}\right)^{1 / 2 s}, \\
l^{2 s} & >\frac{2\left(C_{1}+C_{2}\right) V}{2 r_{1}\left(q-F_{2}\right)}, \\
l^{2 s}\left(l-2 r_{1}\right) & >2 r_{1} l^{2 s} \\
& >\frac{2\left(C_{1}+C_{2}\right) V}{q-F_{2}},
\end{aligned}
$$

where in the last step $l>4 r_{1}$ was used. This gives

$$
\frac{q-F_{2}}{2}>\left(C_{1}+C_{2}\right) V \frac{1}{l^{2 s}} \frac{1}{l-2 r_{1}} .
$$

(iii) From the condition

$$
l>\frac{1+2 F_{2} r_{0} r_{1}+12 F_{2}\left(C_{1}+C_{2}\right) V C_{\infty} C_{a}^{2 s}}{F_{2} r_{0}}
$$


on $l$ we get

$$
r_{0} F_{2} l-2 F_{2} r_{0} r_{1}-12 F_{2}\left(C_{1}+C_{2}\right) V C_{\infty} C_{a}^{2 s}>1 .
$$

By rearranging some terms, we get from the condition

$$
\left(12\left(C_{1}+C_{2}\right) V r_{0}\right)^{1 / 2 s}<l
$$

on $l$ that

$$
\begin{aligned}
12\left(C_{1}+C_{2}\right) V r_{0} & <l^{2 s} \\
& \stackrel{(3.2)}{<} l^{2 s}\left(r_{0} F_{2} l-2 F_{2} r_{0} r_{1}-12 F_{2}\left(C_{1}+C_{2}\right) V C_{\infty} C_{a}^{2 s}\right) \\
& =r_{0} F_{2} l^{1+2 s}-2 F_{2}\left(r_{0} r_{1}+6\left(C_{1}+C_{2}\right) V C_{\infty} C_{a}^{2 s}\right) l^{2 s} \\
l^{1+2 s} r_{0} F_{2}-2 r_{0} r_{1} F_{2} l^{2 s} & <12\left(C_{1}+C_{2}\right) V C_{\infty} F_{2} C_{a}^{2 s} l^{2 s}+12\left(C_{1}+C_{2}\right) V r_{0} \\
\left(C_{1}+C_{2}\right) V \frac{1}{l^{2 s}} \frac{1}{l-2 r_{1}} & <\frac{1}{12} \frac{r_{0} F_{2}}{\left(C_{\infty} C_{a}^{2 s} F_{2} l^{2 s}+r_{0}\right)} .
\end{aligned}
$$

(iv) From definition 3.1 we know that

$$
F_{1}+F_{2}=\left(\frac{\rho}{a-\rho}+1\right) F_{2}=\frac{a}{a-\rho} F_{2} .
$$

Furthermore, from lemma 3.5 we have

$$
\|v\|_{L^{\infty}} \leqslant \frac{2\left(F_{1}+F_{2}\right)}{\pi^{2 s}} \zeta(2 s) a^{2 s-1} \rho .
$$

Putting these together and using (i), we obtain

$$
\begin{aligned}
\|v\|_{L^{\infty}} \leqslant C_{\infty} \frac{a}{a-\rho} F_{2} a^{2 s-1} \rho & <C_{\infty} \frac{a}{\frac{17}{18} a} F_{2} a^{2 s-1} \rho \\
& =\frac{18}{17} C_{\infty} F_{2} a^{2 s-1} \rho \\
& =\frac{18}{17} C_{\infty} F_{2} a^{2 s-1} \frac{1}{3} \frac{a r_{0}}{\left(C_{\infty} F_{2} a^{2 s}+r_{0}\right)} \\
& <\frac{18}{17} C_{\infty} F_{2} a^{2 s-1} \frac{a r_{0}}{3 C_{\infty} F_{2} a^{2 s}} \\
& =\frac{6}{17} r_{0} \\
& <\frac{r_{0}}{2} .
\end{aligned}
$$

(v) Because $F_{1}$ is as in definition 3.1 we have

$$
\begin{aligned}
-F_{1}=-\frac{\rho}{a-\rho} F_{2}<-\frac{\rho}{a} F_{2}<-\frac{\rho / 2}{a} F_{2} & =-\frac{1}{a} \frac{r_{0} a}{6\left(C_{\infty} F_{2} a^{2 s}+r_{0}\right)} F_{2} \\
& \leqslant-\frac{1}{6} \frac{r_{0}}{\left(C_{\infty} C_{a}^{2 s} F_{2} l^{2 s}+r_{0}\right)} F_{2} .
\end{aligned}
$$


Now take $u:=u_{\text {flat }}+u_{\text {lift }}$. Choose the parameters as in lemma 3.24 and

$$
F^{*}:=\frac{1}{2} \min \left\{q-F_{2}, \frac{r_{0}}{6\left(C_{\infty} C_{a}^{2 s} F_{2} l^{2 s}+r_{0}\right)} F_{2}\right\} .
$$

One then has $u \geqslant 0$, as just seen (note that $u_{\text {lift }} \geqslant r_{1}$ and $\left|u_{\text {flat }}\right| \leqslant\|v\|_{L^{\infty}}$ ), and we can now give the proof of theorem 1.3 for $1 / 2<s<1$.

Proof of theorem 1.3 for $1 / 2<s<1$. Let the parameters be as in lemma 3.24 and take $u_{\text {lift }}\left(x_{i}\right)=y_{i}$ for all $i \in I$, which, due to propositions 3.11 and 3.22, is (almost surely) possible. From the choice of $g_{\text {flat }}$ we have

$$
-f\left(x, u_{\text {flat }}(x)+u_{\text {lift }}(x)\right) \leqslant-g_{\text {flat }}(x) .
$$

Using this we have for $F<F^{*}$,

$$
\mathrm{A} u(x)-f(x, u(x, \omega), \omega)+F \leqslant \mathrm{~A} u_{\mathrm{flat}}(x)-g_{\text {flat }}(x)+F+\mathrm{A} u_{\text {lift }}(x) .
$$

With the results of proposition 3.21 and proposition 3.22 it follows that

$$
\begin{aligned}
\mathrm{A} u(x) & -f(x, u(x, \omega), \omega)+F \\
& \leqslant-\min \left\{q-F_{2}, F_{1}\right\}+F+C_{1} \frac{(d / 2+l / 2)^{2-2 s}}{d^{2}} h+C_{2} \frac{h}{(d / 2+l / 2)^{2 s}} \\
& \leqslant-\min \left\{q-F_{2}, F_{1}\right\}+F+\left(C_{1}+C_{2}\right) \frac{h}{l^{2 s}},
\end{aligned}
$$

where in the last step $d=l$ was used. Now applying the estimates of lemma 3.24 and using $h=V /\left(l-2 r_{1}\right)$, we obtain

$$
\begin{aligned}
\mathrm{A} u(x)-f(x, u(x, \omega), \omega)+F \leqslant & -\min \left\{q-F_{2}, \frac{r_{0}}{6\left(C_{\infty} C_{a}^{2 s} F_{2} l^{2 s}+r_{0}\right)} F_{2}\right\} \\
& +F+\left(C_{1}+C_{2}\right) V \frac{1}{l^{2 s}} \frac{1}{l-2 r_{1}} \\
< & -\frac{1}{2} \min \left\{q-F_{2}, \frac{r_{0}}{6\left(C_{\infty} C_{a}^{2 s} F_{2} l^{2 s}+r_{0}\right)} F_{2}\right\}+F^{*} \\
= & 0 .
\end{aligned}
$$

Now picking $\bar{u}$ as in the theorem, we see immediately that the requirements in definition 1.4 are fulfilled: at a point $x$ where $\bar{u}(x, t)$ is already stationary, $\alpha$ must be chosen to be greater than or equal to 0 and the inequality in the definition follows from $(3.3)$. At a point where $\bar{u}(x, t)$ is propagating with velocity $F^{*}$, the parameter $\alpha$ must be chosen to be greater than or equal to $F^{*}$. Note that such a point is necessarily a maximum of $\bar{u}$, so the right-hand side in the definition is bounded from above by $F^{*}$. This concludes the proof that $\bar{u}$ is a supersolution. The estimate on the expected value and the tail distribution of $u(x)$ follows immediately from remark 2.3 and the choice of $p_{H}(1)$ in proposition 3.11 .

For the case in which $s=1 / 2$ some changes in the choice of parameters have to be performed due to the worse $L^{\infty}$-estimate on $v$ in lemma 3.5. 
Lemma 3.25. Let $C_{\rho}=\frac{1}{2} \sqrt{\pi r_{0}^{3} / 48 \mathrm{e}^{2}\left(36 F_{2} / 17 \pi\right)^{3} C_{a}^{3}}$ and choose $C_{a}>5$. Take $q>0$ and $V>0$ as in proposition 3.11. Let $0<F_{2}<q$ and choose $F_{1}>0$ as in definition 3.1. Now choose $l>0$ such that

$$
l>\max \left\{\left(\frac{\left(C_{1}+C_{2}\right) V}{r_{1}\left(q-F_{2}\right)}\right),\left(\frac{2 V\left(C_{1}+C_{2}\right)}{F_{2} C_{\rho}}\right)^{2}+4 r_{1}, 12 \frac{\left(C_{1}+C_{2}\right) C_{a}}{r_{0}} V+2 r_{1}\right\} .
$$

Take $d=l$ and $\frac{3}{2} l=\frac{1}{2} d+l \leqslant a \leqslant C_{a} l$,

$$
\rho=\min \left\{\sqrt{\frac{\pi r_{0}^{3}}{48 \mathrm{e}^{2}\left(36 F_{2} / 17 \pi\right)^{3}}} \frac{1}{\sqrt{a}}, \frac{r_{0}}{3}\right\}
$$

and $h=V /\left(l-2 r_{1}\right)$. We then have:

(i) $l>4 r_{1}$;

(ii) $\rho<\frac{r_{0}}{3}<\frac{a}{18}$;

(iii) $\left(C_{1}+C_{2}\right) V \frac{1}{l} \frac{1}{l-2 r_{1}}<\frac{q-F_{2}}{2}$;

(iv) $\left(C_{1}+C_{2}\right) V \frac{1}{l} \frac{1}{l-2 r_{1}}<\frac{1}{2} F_{2} \min \left\{\frac{1}{2} \sqrt{\frac{\pi r_{0}^{3}}{48 \mathrm{e}^{2}\left(36 F_{2} / 17 \pi\right)^{3} C_{a}^{3}}} \frac{1}{l^{3 / 2}}, \frac{r_{0}}{6 C_{a} l}\right\}$;

(v) $\|v\|_{L^{\infty}}<\frac{r_{0}}{2}$

(vi) $-F_{1} \leqslant-F_{2} \min \left\{\frac{1}{2} \sqrt{\frac{\pi r_{0}^{3}}{48 \mathrm{e}^{2}\left(36 F_{2} / 17 \pi\right)^{3} C_{a}^{3}}}, \frac{1}{l^{3 / 2}}, \frac{r_{0}}{6 C_{a} l}\right\}$.

Proof. (i) Given that $l>\left(2 V\left(C_{1}+C_{2}\right) / F_{2} C_{\rho}\right)^{2}+4 r_{1}$ and $\left(2 V\left(C_{1}+C_{2}\right) / F_{2} C_{\rho}\right)^{2} \geqslant 0$, this is clear.

(ii) From the choice of $\rho$ it is clear that $\rho \leqslant r_{0} / 3$. From the conditions on $r_{0}, r_{1}$ and the choice of $l$, we furthermore have

$$
\frac{r_{0}}{3}<\frac{r_{1}}{3}<\frac{l}{12} \leqslant \frac{2}{3} \frac{a}{12}=\frac{a}{18} .
$$

(iii) From the condition on $l$ (using item (i)) some calculation gives

$$
\begin{gathered}
l>\frac{2\left(C_{1}+C_{2}\right) V}{2 r_{1}\left(q-F_{2}\right)}, \\
l\left(l-2 r_{1}\right)>2 r_{1} l>\frac{2\left(C_{1}+C_{2}\right) V}{q-F_{2}}, \\
\frac{q-F_{2}}{2}>\left(C_{1}+C_{2}\right) V \frac{1}{l} \frac{1}{l-2 r_{1}} .
\end{gathered}
$$

(iv) From the condition

$$
l>\left(\frac{2 V\left(C_{1}+C_{2}\right)}{F_{2} C_{\rho}}\right)^{2}+4 r_{1}
$$


on $l$ one obtains

$$
\begin{aligned}
\left(\frac{2 V\left(C_{1}+C_{2}\right)}{F_{2} C_{\rho}}\right)^{2}<l-4 r_{1}<l-4 r_{1}+\frac{4 r_{1}}{l} & =\frac{l^{2}-4 r_{1} l+4 r_{1}^{2}}{l} \\
& =\frac{\left(l-2 r_{1}\right)^{2}}{l}
\end{aligned}
$$

Therefore, taking the square root, one obtains

$$
\frac{2 V\left(C_{1}+C_{2}\right)}{F_{2} C_{\rho}}<\frac{l-2 r_{1}}{l^{1 / 2}}=\frac{l\left(l-2 r_{1}\right)}{l^{3 / 2}},
$$

which finally, after rearranging and putting in $C_{\rho}$, yields

$$
\left(C_{1}+C_{2}\right) V \frac{1}{l} \frac{1}{l-2 r_{1}}<\frac{1}{2} F_{2} C_{\rho} \frac{1}{l^{3 / 2}}=\frac{1}{2} F_{2} \frac{1}{2} \sqrt{\pi r_{0}^{3} / 48 \mathrm{e}^{2}\left(36 F_{2} / 17 \pi\right)^{3} C_{a}^{3}} \frac{1}{l^{3 / 2}} .
$$

The second part simply follows by rearranging the condition

$$
l>12 \frac{\left(C_{1}+C_{2}\right) C_{a}}{r_{0}} V+2 r_{1}
$$

on $l$ such that

$$
l-2 r_{1}>12 \frac{\left(C_{1}+C_{2}\right) C_{a}}{r_{0}} V
$$

and finally

$$
\left(C_{1}+C_{2}\right) V \frac{1}{l} \frac{1}{l-2 r_{1}}<\frac{1}{2} \frac{r_{0}}{6 C_{a}} \frac{1}{l} .
$$

(v) From lemma 3.5 we have

$$
\|v\|_{L^{\infty}} \leqslant 2 \frac{F_{1}+F_{2}}{\pi} \rho(2+\log (a)-\log (\pi \rho)) .
$$

The choice of $F_{1}$ from definition 3.1 together with item (ii) yields

$$
F_{1}+F_{2}=\left(\frac{\rho}{a-\rho}+1\right) F_{2}=\frac{a}{a-\rho} F_{2} \leqslant \frac{a}{\frac{17}{18} a} F_{2}=\frac{18}{17} F_{2}
$$

Taking both together one gets

$$
\|v\|_{L^{\infty}} \leqslant\left(\frac{36 F_{2}}{17 \pi}\right) \rho(2+\log (a)-\log (\pi \rho)) .
$$

From the choice of $\rho$, we furthermore obtain

$$
\rho \leqslant \min \left\{\sqrt{\frac{\pi r_{0}^{3}}{48 \mathrm{e}^{2}\left(36 F_{2} / 17 \pi\right)^{3}}} \frac{1}{\sqrt{a}}, \frac{r_{0}}{3}\right\}
$$

and therefore, by squaring,

$$
\rho^{2} \leqslant \min \left\{\frac{\pi r_{0}^{3}}{48 \mathrm{e}^{2}\left(36 F_{2} / 17 \pi\right)^{3}} \frac{1}{a}, \frac{r_{0}^{2}}{9}\right\},
$$


so in particular,

$$
\rho^{2} \leqslant \frac{\pi r_{0}^{3}}{48 \mathrm{e}^{2}\left(36 F_{2} / 17 \pi\right)^{3}} \frac{1}{a} .
$$

Using this, by rearranging and adding additional terms we get

$$
\begin{aligned}
\frac{a \mathrm{e}^{2}}{\pi} & \leqslant \frac{1}{48} \frac{r_{0}^{3}}{\left(36 F_{2} / 17 \pi\right)^{3} \rho^{2}} \\
& <\rho\left(1+\frac{r_{0}}{2\left(36 F_{2} / 17 \pi\right) \rho}+\frac{1}{2}\left(\frac{r_{0}}{2\left(36 F_{2} / 17 \pi\right) \rho}\right)^{2}+\frac{1}{6}\left(\frac{r_{0}}{2\left(36 F_{2} / 17 \pi\right) \rho}\right)^{3}\right) \\
& <\rho \exp \left(\frac{r_{0}}{2\left(36 F_{2} / 17 \pi\right) \rho}\right) .
\end{aligned}
$$

In the last step the standard estimate for the series of the exponential function was applied. This shows that we have

$$
a \mathrm{e}^{2}<\pi \rho \exp \left(\frac{r_{0}}{2\left(36 F_{2} / 17 \pi\right) \rho}\right) .
$$

Applying the logarithm gives

$$
2+\log (a)<\log (\pi \rho)+\frac{r_{0}}{2\left(36 F_{2} / 17 \pi\right) \rho}
$$

and this finally yields

$$
\left(\frac{36 F_{2}}{17 \pi}\right) \rho(2+\log (a)-\log (\pi \rho))<\frac{r_{0}}{2} .
$$

(vi) The choice of $F_{1}$ from definition 3.1 and the definition of $\rho$ give

$$
\begin{aligned}
-F_{1}=-\frac{\rho}{a-\rho} F_{2}<-\frac{\rho / 2}{a} F_{2} & =-\frac{1}{2} \frac{F_{2}}{a} \min \left\{\sqrt{\frac{\pi r_{0}^{3}}{48 \mathrm{e}^{2}\left(36 F_{2} / 17 \pi\right)^{3}}} \frac{1}{\sqrt{a}}, \frac{r_{0}}{3}\right\} \\
& =-\frac{F_{2}}{2} \min \left\{\sqrt{\frac{\pi r_{0}^{3}}{48 \mathrm{e}^{2}\left(36 F_{2} / 17 \pi\right)^{3}}} \frac{1}{a^{3 / 2}}, \frac{r_{0}}{3 a}\right\} .
\end{aligned}
$$

As $a \leqslant C_{a} l$ we have $-1 / a \leqslant-1 / C_{a} l$ and therefore

$$
\begin{aligned}
-F_{1} & <-\frac{1}{2} \frac{F_{2}}{a} \min \left\{\sqrt{\frac{\pi r_{0}^{3}}{48 \mathrm{e}^{2}\left(36 F_{2} / 17 \pi\right)^{3}}} \frac{1}{\sqrt{a}}, \frac{r_{0}}{3}\right\} \\
& \leqslant-\frac{F_{2}}{2} \min \left\{\sqrt{\frac{\pi r_{0}^{3}}{48 \mathrm{e}^{2}\left(36 F_{2} / 17 \pi\right)^{3} C_{a}^{3}}} \frac{1}{l^{3 / 2}}, \frac{r_{0}}{3 C_{a} l}\right\} .
\end{aligned}
$$

Now we have everything together to prove the claim for $s=1 / 2$. 
Proof of theorem 1.3 for $s=1 / 2$. Choose the parameters as in lemma 3.25 and define $u:=u_{\text {flat }}+u_{\text {lift }}$ with $u_{\text {lift }}\left(x_{i}\right)=y_{i}$ for all $i \in I$, which, due to propositions 3.11 and 3.22 , is (almost surely) possible. Lemma 3.25 then shows that $u \geqslant 0$ (as $u_{\text {lift }} \geqslant r_{1}$ and $\left.\left|u_{\text {flat }}\right| \leqslant\|v\|_{L^{\infty}}\right)$ and for

$$
F^{*}=\frac{1}{2} \min \left\{q-F_{2}, F_{2} \min \left\{\frac{1}{2} \sqrt{\frac{\pi r_{0}^{3}}{48 \mathrm{e}^{2}\left(36 F_{2} / 17 \pi\right)^{3} C_{a}^{3}}} \frac{1}{l^{3 / 2}}, \frac{r_{0}}{6 C_{a} l}\right\}\right\},
$$

using the estimates of proposition 3.21, proposition 3.22 and lemma 3.25 , we can show, analogously to the proof of the case in which $1 / 2<s<1$, that

$$
\mathrm{A} u(x)-f(x, u(x, \omega), \omega)+F \leqslant 0
$$

for all $F<F^{*}$. The further conclusion also follows as in the case of $1 / 2<s<1$.

Again due to the different $L^{\infty}$-estimate on $v$ for the case in which $s<1 / 2$, the parameters have to be chosen slightly differently.

LEMmA 3.26. Let $0<\alpha<1$ such that $0<2 s-\alpha$ and $C_{a}>5$ fixed.

Choose, furthermore, $q>0$ and $V>0$ as in proposition 3.11. Let $0<F_{2}<q$ be fixed and let $F_{1}>0$ as in definition 3.1 as well as $C_{\rho}=\left(\left(17 \pi / 54 \cdot 2^{2 s} F_{2}\right) s(1-\right.$ $\left.2 s) r_{0}\right)^{1 / 2 s}$. Choose $l>0$ such that

$$
l>\max \left\{4 r_{1},\left(\frac{\left(C_{1}+C_{2}\right) V}{r_{1}\left(q-F_{2}\right)}\right)^{1 / 2 s},\left(\frac{\left(4 r_{1}\right)^{2 s}}{2}+\frac{2 V\left(C_{1}+C_{2}\right) C_{a}}{F_{2} \min \left\{C_{\rho}, r_{0} / 6\right\}}\right)^{1 / 2 s}\right\} .
$$

Choose $d=l$, as well as $\frac{3}{2} l=\frac{1}{2} d+l \leqslant a \leqslant C_{a} l, \rho=2 \min \left\{C_{\rho}, r_{0} / 6\right\}$ and $h=$ $V /\left(l-2 r_{1}\right)$. Then one has:

(i) $l>4 r_{1}$;

(ii) $\rho<\frac{r_{0}}{3}<\frac{a}{18}$;

(iii) $\left(C_{1}+C_{2}\right) V \frac{1}{l^{2 s}} \frac{1}{l-2 r_{1}}<\frac{q-F_{2}}{2}$;

(iv) $\left(C_{1}+C_{2}\right) V \frac{1}{l^{2 s}} \frac{1}{l-2 r_{1}}<\frac{F_{2}}{2 C_{a}} \min \left\{C_{\rho}, \frac{r_{0}}{6}\right\} \frac{1}{l}$;

(v) $\|v\|_{\infty}<\frac{r_{0}}{2}$

(vi) $-F_{1} \leqslant-\frac{F_{2}}{C_{a}} \min \left\{C_{\rho}, \frac{r_{0}}{6}\right\} \frac{1}{l}$.

Proof. (i) This is clear by assumption.

(ii) From the choice of $\rho$ we have $\rho \leqslant r_{0} / 3$. From the assumptions on $r_{0}, r_{1}$ and the choice of $l$ we have, furthermore,

$$
\frac{r_{0}}{3}<\frac{r_{1}}{3}<\frac{l}{12} \leqslant \frac{2}{3} \frac{a}{12}=\frac{a}{18} .
$$


(iii) From the choice of $l$ it follows that

$$
l>\left(\frac{\left(C_{1}+C_{2}\right) V}{r_{1}\left(q-F_{2}\right)}\right)^{1 / 2 s} .
$$

As $l>4 r_{1}$, we furthermore have

$$
\frac{2\left(C_{1}+C_{2}\right) V}{q-F_{2}}<l^{2 s} 2 r_{1}<l^{2 s}\left(l-2 r_{1}\right),
$$

and therefore, finally,

$$
\left(C_{1}+C_{2}\right) V \frac{1}{l^{2 s}} \frac{1}{l-2 r_{1}}<\frac{q-F_{2}}{2} .
$$

(iv) From the choice of $l$ we have

$$
\begin{aligned}
l & >\left(\frac{\left(4 r_{1}\right)^{2 s}}{2}+\frac{2 V\left(C_{1}+C_{2}\right) C_{a}}{F_{2} \min \left\{C_{\rho}, r_{0} / 6\right\}}\right)^{1 / 2 s}, \\
l^{2 s} & >\frac{\left(4 r_{1}\right)^{2 s}}{2}+\frac{2 V\left(C_{1}+C_{2}\right) C_{a}}{F_{2} \min \left\{C_{\rho}, r_{0} / 6\right\}} .
\end{aligned}
$$

Furthermore, we can calculate

$$
l^{2 s}-\frac{\left(4 r_{1}\right)^{2 s}}{2}=l^{2 s}-\frac{2 r_{1}}{4 r_{1}}\left(4 r_{1}\right)^{2 s}=l^{2 s}-\frac{2 r_{1}}{\left(4 r_{1}\right)^{1-2 s}}<l^{2 s}-\frac{2 r_{1}}{l^{1-2 s}},
$$

while in the last step $l>4 r_{1}$ was used. Together, these yield

$$
\frac{2 V\left(C_{1}+C_{2}\right) C_{a}}{F_{2} \min \left\{C_{\rho}, r_{0} / 6\right\}}<l^{2 s}-\frac{\left(4 r_{1}\right)^{2 s}}{2}<l^{2 s}-\frac{2 r_{1}}{l^{1-2 s}}=l^{2 s}\left(l-2 r_{1}\right) \frac{1}{l},
$$

and therefore

$$
\left(C_{1}+C_{2}\right) V \frac{1}{l^{2 s}} \frac{1}{l-2 r_{1}}<\frac{F_{2}}{2 C_{a}} \min \left\{C_{\rho}, \frac{r_{0}}{6}\right\} \frac{1}{l} .
$$

(v) From lemma 3.5 we have the estimate

$$
\|v\|_{L^{\infty}} \leqslant \frac{1}{s(1-2 s) \pi} \rho^{2 s}\left(F_{1}+F_{2}\right) .
$$

From definition 3.1 we have

$$
F_{1}+F_{2}=\frac{a}{a-\rho} F_{2}<\frac{a}{\frac{17}{18} a} F_{2}=\frac{18}{17} F_{2},
$$

where item (ii) was used. Together these yield

$$
\begin{aligned}
\|v\|_{L^{\infty}} \leqslant \frac{1}{s(1-2 s) \pi} \rho^{2 s}\left(F_{1}+F_{2}\right) & \leqslant \frac{18 F_{2}}{17 \pi} \frac{\rho^{2 s}}{s(1-2 s)} \\
& =\frac{18}{17 \pi s(1-2 s)} F_{2} \rho^{2 s} .
\end{aligned}
$$


Together with the definition of $\rho$ we have

$$
\begin{aligned}
\|v\|_{L^{\infty}} \leqslant \frac{18 F_{2}}{17 \pi s(1-2 s)} \rho^{2 s} & \leqslant \frac{18 F_{2}}{17 \pi s(1-2 s)} 2^{2 s}\left(\frac{17 \pi}{54 \times 2^{2 s} F_{2}} s(1-2 s) r_{0}\right) \\
& =\frac{r_{0}}{3} \\
& <\frac{r_{0}}{2} .
\end{aligned}
$$

(vi) From definition 3.1 we have

$$
\begin{aligned}
-F_{1}=-\frac{\rho}{a-\rho} F_{2}<-\frac{\rho / 2}{a} F_{2}=-\frac{F_{2}}{a} \min \left\{C_{\rho}, \frac{r_{0}}{6}\right\} & =-F_{2} \min \left\{C_{\rho}, \frac{r_{0}}{6}\right\} \frac{1}{a} \\
& \leqslant-\frac{F_{2}}{C_{a}} \min \left\{C_{\rho}, \frac{r_{0}}{6}\right\} \frac{1}{l} .
\end{aligned}
$$

Finally, this enables us to give the proof for the case in which $s<1 / 2$.

Proof of theorem 1.3 for $s<1 / 2$. Choose the parameters as in lemma 3.26 and define $u:=u_{\text {flat }}+u_{\text {lift }}$ with $u_{\text {lift }}\left(x_{i}\right)=y_{i}$ for all $i \in I$, which, due to propositions 3.11 and 3.22 , is (almost surely) possible. Lemma 3.25 then shows that $u \geqslant 0$ (as $u_{\text {lift }} \geqslant r_{1}$ and $\left|u_{\text {flat }}\right| \leqslant\|v\|_{L^{\infty}}$ ) and for

$$
F^{*}=\frac{1}{2} \min \left\{q-F_{2}, \frac{F_{2}}{C_{a}} \min \left\{C_{\rho}, \frac{r_{0}}{6}\right\} \frac{1}{l}\right\},
$$

using the estimates of proposition 3.21, proposition 3.22 and lemma 3.26 , we can show, analogously to the proof of the cases in which $s=1 / 2$ and $1 / 2<s<1$, that

$$
\mathrm{A} u(x)-f(x, u(x, \omega), \omega)+F \leqslant 0
$$

for all $F<F^{*}$. Again here, the final conclusion follows as in the case of $1 / 2<s<1$.

\section{Conclusions}

In this paper we have shown the existence of a non-trivial pinning threshold for interfaces in elastic media with local obstacles. Models of the kind discussed frequently arise in physics, for example in the propagation of crack fronts in heterogeneous media. Assuming free propagation of such an interface for large enough driving force (which is trivial to obtain under some conditions on the heterogeneity), we have shown the transition of a microscopically viscous kinetic relation (force $=$ velocity) for interfaces in elastic media with random obstacles to a stick-slip behaviour on larger scales. The construction of the supersolution has been constrained to the $1+1$ dimensional case, i.e. that of a one-dimensional interface propagating in a two-dimensional plane. In many cases, this is the physically relevant situation. The $n$-dimensional case is still open due to technical difficulties mostly concerning the compensation of errors arising from modifying a periodic solution. 
Furthermore, we have shown a percolation result, namely, a non-trivial percolation threshold for the existence of an infinite cluster in next-nearest neighbour site percolation that is the graph of an only logarithmically growing function.

\section{Acknowledgements}

P.W.D. and M.S. gratefully acknowledge support from the DFG research unit FOR 718 'Analysis and Stochastics in Complex Physical Systems'. We thank the anonymous referees for their comments.

\section{References}

1 A. Briani and R. Monneau. Time-homogenization of a first order system arising in the modelling of the dynamics of dislocation densities. C. R. Math. 347 (2009), 231-236.

2 L. Caffarelli and L. Silvestre. An extension problem related to the fractional Laplacian. Commun. PDEs 32 (2007), 1245-1260.

3 E. Di Nezza, G. Palatucci and E. Valdinoci. Hitchhiker's guide to the fractional Sobolev spaces. Bull. Sci. Math. 136, 521-573.

4 N. Dirr, P. W. Dondl, G. R. Grimmett, A. E. Holroyd and M. Scheutzow. Lipschitz percolation. Electron. Commun. Prob. 15 (2010), 14-21.

$5 \quad$ N. Dirr, P. W. Dondl and M. Scheutzow. Pinning of interfaces in random media. Interfaces Free Boundaries 13 (2011), 411-321.

6 J. Droniou and C. Imbert. Fractal first-order partial differential equations. Arch. Ration. Mech. Analysis 182 (2006), 299-331.

7 D. Ertas and M. Kardar. Critical dynamics of contact line depinning. Phys. Rev. E 49 (1994), R2532-R2535.

8 N. Forcadel, C. Imbert and R. Monneau. Homogenization of some particle systems with two-body interactions and of the dislocation dynamics. Discrete Contin. Dynam. Syst. A 23 (2009), 785-826.

9 H. Gao and J. R. Rice. A first-order perturbation analysis of crack trapping by arrays of obstacles. J. Appl. Mech. 56 (1989), 828-836.

10 G. R. Grimmett and A. E. Holroyd. Geometry of Lipschitz percolation. Annales Inst. H. Poincaré B 48 (2012), 309-326.

11 C. Imbert. A non-local regularization of first order Hamilton-Jacobi equations. J. Diff. Eqns 211 (2005), 218-246.

12 J. F. Joanny and P. G. de Gennes. A model for contact angle hysteresis. J. Chem. Phys. 81 (1984), 552-562.

13 S. Moulinet, C. Guthmann and E. Rolley. Roughness and dynamics of a contact line of a viscous fluid on a disordered substrate. Eur. Phys. J. E 8 (2002), 437-443.

14 J. Schmittbuhl, A. Delaplace, K. J. Maloy, H. Perfettini and J. P. Vilotte. Slow crack propagation and slip correlations. Pure Appl. Geophys. 160 (2003), 961-976.

15 A. Tanguy and T. Vettorel. From weak to strong pinning. I. A finite size study. Eur. Phys. $J$. B 38 (2004), 71-82.

(Issued 5 June 2015) 